\title{
Fault-Tolerant Continuous-Variable Measurement-based Quantum Computation Architecture
}

\author{
Mikkel V. Larsen $\odot,{ }^{1, *}$ Christopher Chamberland $\odot, 2,3, \ddagger$ Kyungjoo Noh $\odot, 2,3, \ddagger$ \\ Jonas S. Neergaard-Nielsen $\odot,{ }^{1}$ and Ulrik L. Andersen ${ }^{1, \dagger}$ \\ ${ }^{1}$ Department of Physics, Center for Macroscopic Quantum States (bigQ), Technical University of Denmark, \\ Fysikvej, 2800 Kgs., Lyngby, Denmark \\ ${ }^{2}$ AWS Center for Quantum Computing, Pasadena, California 91125, USA \\ ${ }^{3}$ IQIM, California Institute of Technology, Pasadena, California 91125, USA
}

(Received 11 January 2021; revised 18 May 2021; accepted 23 July 2021; published 11 August 2021)

\begin{abstract}
Continuous-variable measurement-based quantum computation on cluster states has in recent years shown great potential for scalable, universal, and fault-tolerant quantum computation when combined with the Gottesman-Kitaev-Preskill (GKP) code and quantum error correction. However, no complete fault-tolerant architecture exists that includes everything from cluster-state generation with finite squeezing to gate implementations with realistic noise and error correction. In this work, we propose a simple architecture for the preparation of a cluster state in three dimensions in which gates can be efficiently implemented by gate teleportation. To accommodate scalability, we propose architectures that allow both spatial and temporal multiplexing, with the temporally encoded version requiring as little as two squeezed light sources. Because of its three-dimensional structure, the architecture supports topological qubit error correction, while GKP error correction is efficiently realized within the architecture by teleportation. To validate fault tolerance, the architecture is simulated using surface-GKP codes, including noise from GKP states as well as gate noise caused by finite squeezing in the cluster state. We find a fault-tolerant squeezing threshold of $12.7 \mathrm{~dB}$, with room for further improvement.
\end{abstract}

DOI: 10.1103/PRXQuantum.2.030325

\section{INTRODUCTION}

In measurement-based quantum computation (MBQC), gates are implemented by projective measurements on a multimode entangled cluster state, circumventing the complex coherent unitary dynamics required in conventional gate-based quantum computation [1]. As such, the cluster state is a critical resource for MBQC, and its number of modes and structural design define the size of a potential measurement-induced algorithm. A particularly promising platform for scaling and controlling the structure of a cluster state is the optical-continuous-variable (CV) platform [2,3], where large cluster states can be deterministically generated and controlled, and efficiently

\footnotetext{
*mikkel.vilsboell@gmail.com

†ulrik.andersen@fysik.dtu.dk

¥This work was done before $\mathrm{CC}$ and $\mathrm{KN}$ joined the AWS Center for Quantum Computing.

Published by the American Physical Society under the terms of the Creative Commons Attribution 4.0 International license. Further distribution of this work must maintain attribution to the author(s) and the published article's title, journal citation, and DOI.
}

measured by homodyne detection. This has been proven by realizations of large-scale CV cluster states in both one dimension [4-6] and two dimensions [7,8]. Moreover, the versatility of the $\mathrm{CV}$ optical platform has been further corroborated by recent demonstrations of single-mode and multimode gates using high-efficiency projective measurements on one-dimensional [9] and two-dimensional [10] cluster states.

MBQC based on the CV platform is, however, inherently noisy due to the impossibility of generating maximally entangled $\mathrm{CV}$ cluster states: the generation of maximal $\mathrm{CV}$ entanglement requires squeezed states of infinite squeezing and thereby infinite energy, which is not feasible. Therefore, inevitably, Gaussian noise is added to the quantum information during computation. To combat this additive noise, information is encoded as special qubits in $\mathrm{CV}$ bosonic modes of infinite dimension. By encoding such qubits into the bosonic modes, using, e.g., a cat-code [11], a binomial code [12], or the GottesmanKitaev-Preskill (GKP) code [13], this Gaussian noise can be corrected at the cost of being converted into Pauli errors in the encoded qubit. These Pauli qubit errors must then be corrected by some qubit quantum error-correction scheme. Implementing qubit error correction efficiently 
in MBQC puts stringent requirements on the underlying cluster state. As an example, the local connectivity in the cluster states supports only coupling between nearestneighbor modes, so topological error correction is a natural choice for qubit error correction [14]. This, in turn, requires a three-dimensional (3D) cluster state for MBQC [15-17].

Several different proposals for 3D cluster-state generation and topological MBQC exist. Fukui et al. [18] suggested a scheme for fault-tolerant MBQC based on topological error correction, but their scheme assumes the availability of a highly complex 3D cluster state of encoded qubits. Wu et al. [19] proposed an optical setup for the generation of a $3 \mathrm{D}$ cluster state using time and frequency multiplexing. However, in their proposal, gates are implemented by gate teleportation through four-mode square cluster states, leading to increased gate noise. In another publication by Fukui et al. [20], an all-temporally encoded 3D cluster state was proposed, but this scheme is experimentally highly challenging, as it requires the construction of 12 squeezing sources and real-time feedforward operations. Moreover, no schemes for qubit encoding and qubit error correction were put forward. The most complete work on CV MBQC to date has been carried out by Bourassa et al. [21], in which a computational architecture for the generation of a 3D cluster state combined with topological MBQC is proposed. However, the suggested architecture is based on spatial encoding, rendering the amount of spatial resources required very large (as this number scales linearly with the computation size). Moreover, their scheme relies on a very large number of experimentally challenging online swap and sum gates, which they assume to be ideal. Experimental work towards topological quantum computation has been demonstrated on other platforms, including a nine-qubit code on a photonic platform with polarization-encoded qubits [22], and a seven-qubit code on an ion-trap platform [23] and a superconducting platform [24]. Still, thousands of qubits are required for large fault-tolerant codes [25].

In our work, we propose a simple, scalable, and complete architecture for topological MBQC and validate the fault tolerance of the computation scheme. The scheme is based on gate teleportation on parallel one-dimensional (1D) cluster states, or wires, arranged in a 3D lattice and coupled by variable beam splitters for two-mode gates. As such, the setup is a variation of the well-demonstrated 1D cluster-state generation $[4,7,9]$ with added variable beam splitters. Combined with GKP-encoded qubits [13], the scheme allows universal computation, while fault tolerance is achievable by encoding logical qubits in a topological surface code [25-27]. Furthermore, the scheme, being based on gate teleportation, is compatible with a recently proposed GKP correction protocol that dispenses with demanding coupling to ancillary GKP qubits [28]. We validate the fault tolerance of the full scheme by a thorough simulation that includes both noise in the GKP qubits and, unlike previous work, gate noise caused by finite squeezing in the cluster state. As a result, when the topological surface code is combined with GKP error correction in the surface-GKP code [29], we find a squeezing threshold of $17.3 \mathrm{~dB}$. We continue by proposing a variation of the surface-GKP code - the surface-4-GKP code, with four GKP corrections during the surface-code syndrome measurements - by means of which we upgrade the squeezing threshold to $12.7 \mathrm{~dB}$ while leaving room for further improvement. Related schemes in Refs. [18,21] have better thresholds, but they assume an ideal cluster state, i.e., without gate noise. We obtain a comparable threshold of $10.2 \mathrm{~dB}$ when we ignore gate noise.

The paper is organized as follows. In Sec. II, we present the computation scheme and describe the implementation of the required gates. In Sec. III, we focus on GKP error correction within the computation scheme, and in Sec. IV we present the implementation of the surface code for qubit error correction and our validation of the faulttolerance properties by performing simulations. In Sec. VI, we discuss the results and conclude the paper.

\section{COMPUTATION SCHEME}

The concept of our computation scheme is illustrated in Fig. 1. The scheme consists of parallel wires, each corresponding to temporally encoded one-dimensional cluster states $[4,6]$ on which single-mode gates can be implemented by projective measurements using a beam splitter

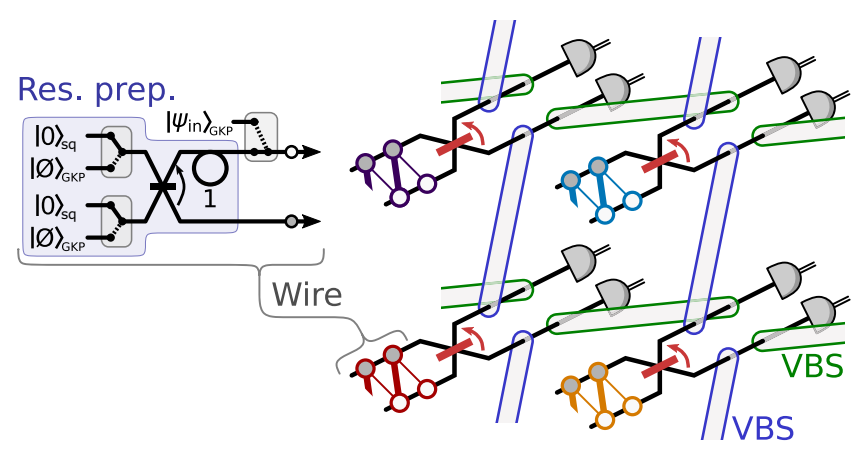

FIG. 1. Conceptual illustration of the computation scheme. $n \times m$ wires of two-mode entangled states are prepared in $n \times m$ resource-preparation gadgets ("Res. prep."). States with input information for computation, $\left|\psi_{\mathrm{in}}\right\rangle_{\mathrm{GKP}}$, are switched into each wire for computation using an optical switch. With a beam splitter (marked with a red arrow) and two detectors for each wire, single-mode gates are implemented in each wire when the resource-preparation gadget is fed with squeezed states, $|0\rangle_{\mathrm{sq}}[9,30]$. Feeding the resource-preparation gadget with special GKP states, namely qunaught states, $|\varnothing\rangle_{\mathrm{GKP}}$, allows GKP quadrature correction of encoded GKP qubits [28]. Neighboring wire setups are connected using variable beam splitters (VBSs), allowing tunable coupling for implementing two-mode gates and thereby enabling multimode computation. 

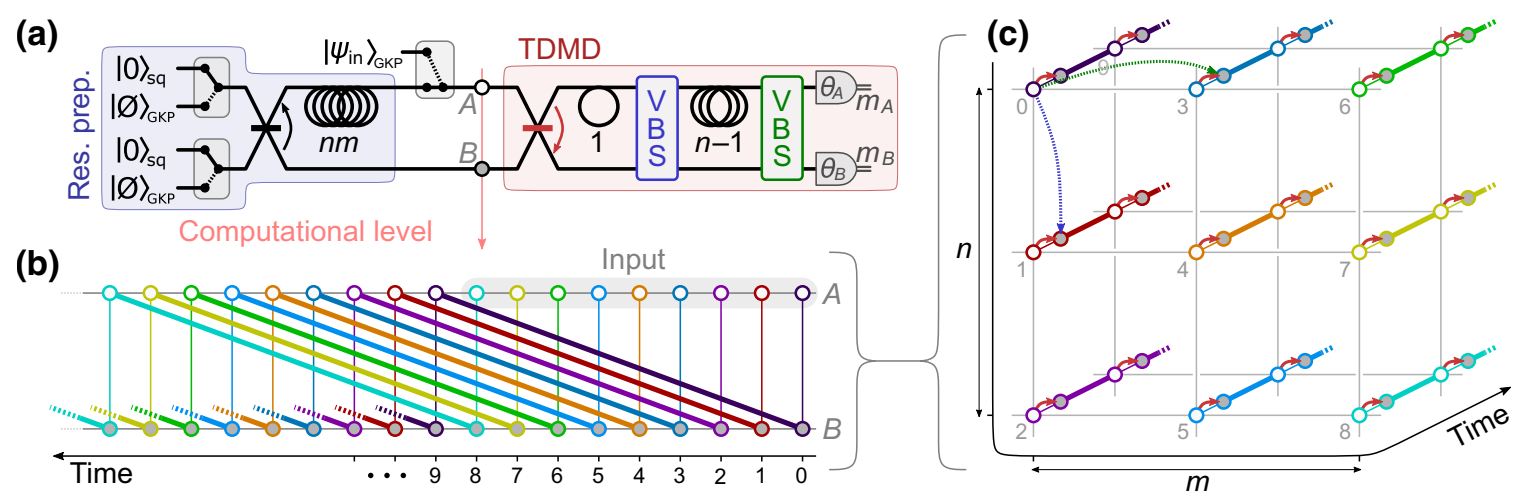

FIG. 2. (a) Temporally encoded computational setup for our scheme. The scheme consists of three parts: the resource-preparation gadget; the computational level, where the computation takes place; and the temporally delocalized measurement device (TDMD) for gate implementation by projective measurements. This scheme utilizes temporal multiplexing of two spatial modes, $A$ and $B$, marked in the computational level. (b) Wires of two-mode entanglement at the computational level shown in the time domain, here for the simple case of $n m=9$. The bold lines represent two-mode entanglement, while the thin lines indicate the temporal overlap of $A$ and $B$. The wires begin with $\mathrm{nm}$ input states in temporal modes 0 to 8 , switched in using an optical switch in $A$ in the computational level. The colors of the wires have no physical meaning and are used merely to indicate different wires. (c) Wires rearranged into a 3D time lattice, where the input is encoded onto the $n \times m$ end surface, while gates are implemented by teleportation along the wires in the third dimension. Here, the red arrows represent the first beam splitter of the TDMD, while the dotted blue and green arrows represent the variable beam splitters of the TDMD. The first ten temporal modes in (b) from 0 to 9 are labeled in (c).

(marked in Fig. 1 with a red arrow) and two detectors [9,30]. Input states, $\left|\psi_{\text {in }}\right\rangle$, can be swapped into each wire for computation using an optical switch. To enable multimode computation, the setups of neighboring wires are connected with variable beam splitters, which allow tunable coupling of wires for implementing two-mode gates. This architecture may be implemented spatially as depicted in Fig. 1, with a large grid of wire setups forming a 3D cluster state encoded in $(\text { space })^{2} \times$ time. Such a spatial encoding requires spatially scalable resources and may be possible with integrated photonics. As an alternative, in Fig. 2 we propose an all-temporally encoded version of the computation scheme that allows a simple experimental implementation and easy scalability. In the following, while we focus on the temporally encoded architecture when describing the computation scheme in detail, all the methods, results, and conclusions presented also hold true for the spatially encoded architecture - even a combination of the spatial and temporal encoding architectures might lead to a similar computation scheme with identical conclusions.

The temporally encoded scheme in Fig. 2 consists of three parts: the preparation of resource states, the injection of input states at the computational level, and the measurements, enabled by a temporally delocalized measurement device. Note that the term "computational level" refers to the location in the setup at which information is encoded and computation takes place. In some of our previous work, this computational level was referred to as the "logic level" [10,31]. However, in the present work we reserve the term "logic" for the qubit error correction presented in Sec. IV. As ancillary input for the resource preparation, we switch between squeezed vacuum states, $|0\rangle_{\text {sq }}$, when implementing gates by projective measurements, and GKP qunaught states [28], $|\varnothing\rangle_{\mathrm{GKP}}$, when performing GKP error correction. In Sec. III, GKP error correction with ancillary $|\varnothing\rangle_{\mathrm{GKP}}$ states is described, while throughout this section we focus on gate implementation with ancillary $|0\rangle_{\text {sq }}$ states.

In the resource-preparation stage, the spatial modes $A$ and $B$ are initially occupied by squeezed vacuum states, $|0\rangle_{\text {sq }}$, which are squeezed along the orthogonal quadratures $(\hat{q}-\hat{p}) / \sqrt{2}$ and $(\hat{q}+\hat{p}) / \sqrt{2}$, respectively. Here, $\hat{q}$ and $\hat{p}$ are the electric field amplitude and phase (or position and momentum) quadratures, for which we use the $\hbar=1$ convention, corresponding to a vacuum variance of $1 / 2$. Each pair of squeezed states is then interfered on a balanced beam splitter, leading to two-mode entanglement with $\hat{q} \hat{p}$ correlations. This is an approximate cluster state equivalent to a conventional two-mode squeezed state (with $\hat{q} \hat{q}$ and $\hat{p} \hat{p}$ correlations) that is phase-rotated by $\pi / 4$ in both modes $[32,33]$. As a unitary operator for the balanced beam splitter, we use $\hat{B}=e^{-i \pi\left(\hat{q}_{i} \otimes \hat{p}_{j}-\hat{p}_{i} \otimes \hat{q}_{j}\right) / 4}$, with the corresponding symplectic matrix

$$
\mathbf{B}=\frac{1}{\sqrt{2}}\left(\begin{array}{cccc}
1 & -1 & 0 & 0 \\
1 & 1 & 0 & 0 \\
0 & 0 & 1 & -1 \\
0 & 0 & 1 & 1
\end{array}\right)
$$

acting on quadrature vectors $\left(\hat{q}_{i}, \hat{q}_{j}, \hat{p}_{i}, \hat{p}_{j}\right)^{T}$, represented graphically by an arrow pointing from mode $i$ to $j$. Note that in this work we prepare two-mode cluster states; however, we could just as well have considered preparation of 
conventional two-mode squeezed states (with $\hat{q} \hat{q}$ and $\hat{p} \hat{p}$ correlations), which are equivalent to cluster states under phase rotation that may be absorbed into the measurement bases.

After interference at the beam splitter, the modes of $A$ are delayed by $\mathrm{nm}$ temporal modes, leading to synchronization of the modes $A$ and $B$ of two-mode entangled states that initially are separated by $\mathrm{nm}$ temporal modes [34]. The result is $\mathrm{nm}$ decoupled wires of two-mode entangled states, illustrated in the time domain in Fig. 2(b) for $n m=9$. Here, each color indicates a different wire, with the bold lines indicating two-mode entanglement, while the thin lines indicate temporal overlap of $A$ and $B$. The $\mathrm{nm}$ wires constitute the computational level, in which computation is performed. Using an optical switch, $\mathrm{nm}$ input modes to the computation can be switched into the computational level at $A$-optical switching has been demonstrated in a continuous-variable quantum setting in Refs. [35,36]. In Fig. 2(b), nine input modes are switched into $A$ in temporal modes 0 to 8 .

The $n m$ wires can be arranged in a $2 \mathrm{D}$ grid such that they form a 3D square lattice, as shown in Fig. 2(c). The third dimension is, in principle, arbitrarily deep. As such, information is encoded on a surface, while computation proceeds along the third dimension by teleportation using the TDMD. The TDMD consists of a balanced beam splitter, two VBSs, two delays of 1 and $n-1$ temporal modes, and two homodyne detectors (HDs) measuring $A$ and $B$ in bases $\hat{q}(\theta)=\hat{q} \cos \theta+\hat{p} \sin \theta$. The arrangement is illustrated in Fig. 2(a). Each VBS can vary between two settings: when single-mode gates are implemented, the VBSs are left "open" such that the modes $A$ and $B$ do not interfere, corresponding to $\hat{I}_{A} \otimes \hat{I}_{B}$; when twomode gates are implemented, one of the two VBSs is "enabled" to function as a balanced beam splitter, with the symplectic matrix in Eq. (1) interfering $A$ and $B$. Such a variable beam splitter may be implemented in various ways, for instance as a Mach-Zehnder interferometer with a controllable phase in one arm, or by polarization control combined with polarization-dependent beam splitters [36-38].

When the VBSs are left open, the TDMD simply implements a two-mode joint Bell measurement, which enacts a single-mode gate teleportation through the two-mode entangled resource state [9,30]. The state in the computation is teleported from temporal mode $k$ in $A,(A, k)$, to mode $(A, k+n m)$. In this process, depending on the HD basis settings $\theta_{A, k}$ and $\theta_{B, k}$, the gate operation

$$
\hat{R}\left(\theta_{+}\right) \hat{S}\left(\tan \theta_{-}\right) \hat{R}\left(\theta_{+}\right)
$$

is implemented on the teleported state, where $\theta_{ \pm}=$ $\left( \pm \theta_{A, k}+\theta_{B, k}\right) / 2$ [39]. Here $\hat{R}(\theta)=e^{-i \theta\left(\hat{q}^{2}+\hat{p}^{2}\right) / 2}$ and
$\hat{S}(s)=e^{i \ln (s)(\hat{q} \hat{p}+\hat{p} \hat{q}) / 2}$ are the rotation and squeezing operators. Note that $k$ labels the temporal modes at the computational level, while at the HDs, modes in $A$ are delayed by $n$ temporal modes relative to modes in $B$. All single-mode Gaussian gates can be implemented with two iterations of Eq. (2) [40].

By enabling one of the two VBSs, two-mode gates can be implemented between nearest neighbors in the 3D time lattice. Two-mode gates between $(A, k)$ and $(A, k+1)$ are implemented by enabling the first VBS, while enabling the second VBS allows two-mode gates between $(A, k)$ and $(A, k+n)$. In the 3D time lattice in Fig. 2(c), the VBSs are represented by dotted arrows. To encode the surface code described in Sec. IV, we implement two different symmetric two-mode gates: $\hat{C}_{Z}(g)=e^{i g \hat{q} \otimes \hat{q}}$ and $\hat{C}_{X}(g)=e^{-i g \hat{p} \otimes \hat{p}}$. These are controlled-phase gates that displace one mode in $\hat{p}$ (or $\hat{q}$ ) by an amount $g \hat{q}$ (or $g \hat{p}$ ), controlled by the other mode. We note that $\hat{C}_{X}(g)$ does not correspond to a controlled-NOT gate. $\hat{C}_{Z}(g)$, or $\hat{C}_{X}(g)$, constitutes together with Eq. (2) a universal Gaussian gate set. In practice, $\hat{C}_{Z}(g)$ and $\hat{C}_{X}(g)$ cannot be implemented in a single computation step without some Fourier by-products of $\pi / 2$ phase rotations, $\hat{F}=\hat{R}(\pi / 2)$. To implement the surface code in Sec. IV with a minimum number of computation steps, we make use of four variations of $\hat{C}_{Z}(g)$ and $\hat{C}_{X}(g)$ with different by-products, each listed in Table I with the basis settings required for implementation. These have by-products of $\hat{F} \otimes \hat{F}^{\dagger}$ or $\hat{F}^{\dagger} \otimes \hat{F}$ when implemented on modes $(A, k) \otimes(A, k+j)$, where $j=1$ or $n$ depending on which VBS is enabled. When the surface code is implemented, the gates are arranged such that the Fourier by-products cancel.

TABLE I. Two-mode gates with input and output in modes $(A, k) \otimes(A, k+j)$ and $(A, k+n m) \otimes(A, k+n m+j)$, respectively, and their required basis settings. Here, $j=1$ when the first VBS (marked by blue in Fig. 2) is enabled, and $j=n$ when the second VBS (marked by green in Fig. 2) is enabled. The order of the tensor products is arranged with earlier temporal modes first. Note that, as apparent from the basis settings, $\left(\hat{F}^{\dagger} \otimes \hat{F}\right) \hat{C}_{Z}(g)=$ $\hat{C}_{X}(g)\left(\hat{F}^{\dagger} \otimes \hat{F}\right)$ and $\hat{C}_{Z}(g)\left(\hat{F} \otimes \hat{F}^{\dagger}\right)=\hat{C}_{X}(g)\left(\hat{F}^{\dagger} \otimes \hat{F}\right)$. However, when the gates are implemented in the surface code in Sec. IV, it is useful to consider them individually, as we have Fourier by-products to cancel.

\begin{tabular}{lc}
\hline \hline Two-mode gate & Basis setting, $\left(\theta_{A, k}, \theta_{B, k}, \theta_{A, k+j}, \theta_{B, k+j}\right)$ \\
\hline$\left(\hat{F}^{\dagger} \otimes \hat{F}\right) \hat{C}_{Z}(g)$ & $\left(-\arctan \frac{2}{g}, 0,0, \arctan \frac{2}{g}\right)$ \\
$\hat{C}_{Z}(g)\left(\hat{F} \otimes \hat{F}^{\dagger}\right)$ & $\left(-\frac{\pi}{2}+\arctan \frac{2}{g}, \frac{\pi}{2},-\frac{\pi}{2}, \frac{\pi}{2}-\arctan \frac{2}{g}\right)$ \\
$\left(\hat{F} \otimes \hat{F}^{\dagger}\right) \hat{C}_{X}(g)$ & $\left(-\frac{\pi}{2}+\arctan \frac{2}{g}, \frac{\pi}{2},-\frac{\pi}{2}, \frac{\pi}{2}-\arctan \frac{2}{g}\right)$ \\
$\hat{C}_{X}(g)\left(\hat{F}^{\dagger} \otimes \hat{F}\right)$ & $\left(-\arctan \frac{2}{g}, 0,0, \arctan \frac{2}{g}\right)$ \\
\hline \hline
\end{tabular}


Finally, as the resource squeezed states $|0\rangle_{\text {sq }}$ are finitely squeezed, all gate implementations inevitably produce excess noise, which accumulates in the computational modes throughout the computation. Because of the Gaussian nature of the quadrature distribution of $|0\rangle_{\mathrm{sq}}$, this gate noise leads to a Gaussian convolution of the quadratures of all computational modes [10,30,31]. Assuming the variance of the squeezed quadrature of $|0\rangle_{\mathrm{sq}}$ to be $\sigma^{2}=e^{-2 r} / 2$ (where $r$ is the squeezing parameter), the variance of the uncorrelated gate noise is

$$
\sigma_{\text {gate }}^{2}=2 \sigma^{2}=e^{-2 r}
$$

which is added symmetrically in each quadrature of the computational modes. In addition to gate noise, an implemented gate also results in a displacement of the computational modes depending on the outcomes of the projective measurements. Since the measurement outcomes are known, this displacement can be compensated for by another cancelling displacement operation. However, in practice, these ubiquitous displacement operations need not be executed directly on the output modes; they can simply be accounted for in postprocessing of the measurement outcomes. Therefore, in this work, we ignore these displacements, while for practical implementation, one has to keep these in mind when analysing the measurement outcomes.

For a derivation of implemented gates considered in this section, together with their resulting gate noise and displacements, see Appendix A.

\section{GKP QUADRATURE CORRECTION}

As mentioned above, noise is added to the computation modes at each gate implementation due to the finite amount of squeezing of the resource states. To correct for this noise and thus prevent noise accumulation, we consider a quadrature noise-correction scheme that relies on bosonic qubit encoding in the infinite-dimensional Hilbert space. This noise-correction scheme, however, comes at the cost of introducing qubit errors, which must be subsequently corrected by a qubit error-correction scheme. The first correction layer, the quadrature correction scheme, is discussed in this section, while the second correction layer, the qubit error correction, is the subject of Sec. IV.

Several schemes for encoding qubits into bosonic harmonic oscillators of infinite Hilbert-space dimension exist, including cat-codes [11], binomial codes [12], and the GKP code [13]. Since the gate noise in our computation scheme is additive quadrature noise, GKP encoding, where a qubit is encoded in the mode quadratures as Dirac combs, is most suitable. The GKP code is also suitable for correcting excitation-loss errors, since excitation loss can be converted via quantum-limited amplification into additive quadrature noise [41-44]. Furthermore, as the gate noise (with its variance given in Eq. (3)) is added symmetrically in phase space, we consider GKP qubits encoded on square grids in phase space with a $2 \sqrt{\pi} \times 2 \sqrt{\pi}$ unit cell. For such encoded qubits, a universal Clifford gate set is realized by the Gaussian gates $\left\{\hat{R}(\pi / 2), \hat{P}(1), \hat{C}_{Z}(1)\right\}$ together with $\sqrt{\pi}$ displacements in phase space. For a comprehensive review of the GKP code, see Refs. $[13,45,46]$.

Information encoded in GKP qubit states, $\left|\psi_{\text {in }}\right\rangle_{\mathrm{GKP}}$, is launched into the computation scheme at the computational level as shown in Fig. 2(a). These states are not ideal, as they are subject to finite-energy constraints (similarly to the squeezed states). This means that the uncertainties in the individual spikes of the quadrature comb in the GKP state are not zero but have a finite value. Mathematically, the delta functions of the Dirac comb in the GKP-state quadrature wave function are replaced by finitely squeezed Gaussian functions, each with a variance of $\sigma_{\mathrm{GKP}}^{2}$, such that the $\hat{q}$-quadrature wave functions of the approximate GKP Pauli- $Z$ eigenstates, $\left|\bar{j}_{\text {GKP }}\right\rangle$ (where $j=0,1$ ), are

$\psi_{j}(q) \propto E(q) \sum_{n=-\infty}^{\infty} \exp \left[\frac{-[q-(2 n+j) \sqrt{\pi}]^{2}}{2 \sigma_{\mathrm{GKP}}^{2}}\right], j=0,1$.

Here $E(q)$ is an overall envelope that can be chosen to satisfy the Fourier relations between the orthogonal $\hat{q}$ and $\hat{p}$ quadratures with equally squeezed spikes - different finitely squeezed approximations of GKP states exist with different envelopes [47]. In the following, for the sake of simplifying the simulation of the fault-tolerance squeezing threshold in Sec. IV, we ignore the overall envelope, i.e., we set $E(q)=1$. Doing so corresponds to a noisier GKP state that is an incoherent mixture of ideal GKP states. This is therefore a conservative assumption that does not lead to false positive results in the noise model [29]. Moreover, for the small $\sigma_{\mathrm{GKP}}$ values considered in this work, the envelopes are correspondingly broad, and we expect that ignoring these will have little effect on the simulated error thresholds presented here. We further assume that the squeezing of the GKP spikes in both the $\hat{q}$ and the $\hat{p}$ quadratures is the same as that of the $|0\rangle_{\mathrm{sq}}$ states in the resource preparation,

$$
\sigma_{\mathrm{GKP}}^{2}=\sigma^{2}=e^{-2 r} / 2
$$

The Gaussian noise accompanying gate implementation results in the variance of the GKP spikes increasing by $\sigma_{\text {gate }}^{2}$ in both quadratures for every single gate. To prevent this, GKP quadrature correction is performed, preferably after every gate. Traditionally, this is done by coupling each quadrature to ancillary GKP states, which are then measured, and the result is fed forward to displacements of the 
computational qubit (or compensated for in following measurement outcomes) [13]. However, this on-demand coupling of desired modes with encoded GKP qubits to ancillary GKP states requires either active squeezing, which is experimentally hard to realize, or projective measurements, which add noise. Instead, we use a new approach presented by Walshe et al. [28], where GKP quadrature correction is realized by qubit teleportation using ancillary GKP qunaught states and is directly compatible with our computation scheme. The GKP qunaught state, $|\varnothing\rangle_{\mathrm{GKP}}$, is the one-level version of the generalized GKP qudit state with a $\sqrt{2 \pi}$ spacing between the spikes in the quadrature wave functions $[28,48]$ :

$$
\psi_{\varnothing}(q) \propto \sum_{n=-\infty}^{\infty} \exp \left[\frac{-(q-n \sqrt{2 \pi})^{2}}{2 \sigma_{\mathrm{GKP}}^{2}}\right],
$$

where the overall envelope is ignored as well, and we assume the spike squeezing in both quadratures to equal that of the GKP qubit states with variance $\sigma_{\mathrm{GKP}}^{2}$. As such, $|\varnothing\rangle_{\mathrm{GKP}}$ holds no information, but interfering two $|\varnothing\rangle_{\mathrm{GKP}}$ states on a beam splitter results in a two-mode GKP-qubit Bell state - for more information, see Ref. [28]. This state can then be used for GKP-qubit teleportation with support only on the GKP grid in phase space, so that a noisy GKP qubit is projected into a purified GKP qubit by the teleportation.

The implementation of the GKP quadrature correction in Ref. [28] is shown in Fig. 3. In the resource-preparation gadget, we switch from $|0\rangle_{\mathrm{sq}}$ to $|\varnothing\rangle_{\mathrm{GKP}}$ states. After interference on the first beam splitter, a GKP Bell state is prepared at the computational level instead of a two-mode $\mathrm{CV}$ cluster state. For teleportation of a noisy GKP qubit through the GKP Bell state, a Bell measurement of the noisy GKP qubit and one mode of the Bell state needs to be carried out by the TDMD. This is done by leaving the two VBSs open and measuring in the $\hat{q}$ and $\hat{p}$ bases in spatial modes $A$ and $B$, respectively. The corresponding graph in a small section of the $3 \mathrm{D}$ time lattice is shown in Fig. 3 together with the corresponding circuit. The resulting Kraus operator,

$$
\hat{K}\left(m_{A}, m_{B}\right)=\mathcal{N} \hat{\bar{\Pi}}_{\mathrm{GKP}} \hat{X}\left(-m_{A} \sqrt{2}\right) \hat{Z}\left(-m_{B} \sqrt{2}\right),
$$

projects the noisy input state into a purified GKP qubit state. Here, $\hat{X}\left(-m_{A} \sqrt{2}\right)=e^{i m_{A} \sqrt{2} \hat{p}}$ and $\hat{Z}\left(-m_{B} \sqrt{2}\right)=$ $e^{-i m_{B} \sqrt{2} \hat{q}}$ are displacements in the $\hat{q}$ and $\hat{p}$ quadratures, respectively, depending on the measurement outcomes $m_{A}$ and $m_{B} ; \mathcal{N}$ is a normalization factor, also depending on the measurement outcomes; and

$$
\hat{\bar{\Pi}}_{\mathrm{GKP}}=\left|\overline{0}_{\mathrm{GKP}}\right\rangle\left\langle\overline{0}_{\mathrm{GKP}}|+| \overline{1}_{\mathrm{GKP}}\right\rangle\left\langle\overline{1}_{\mathrm{GKP}}\right|
$$

is a noisy GKP projector (here, the $\left|\bar{j}_{\text {GKP }}\right\rangle$ are the approximate GKP Pauli- $Z$ eigenstates for the quadrature wave

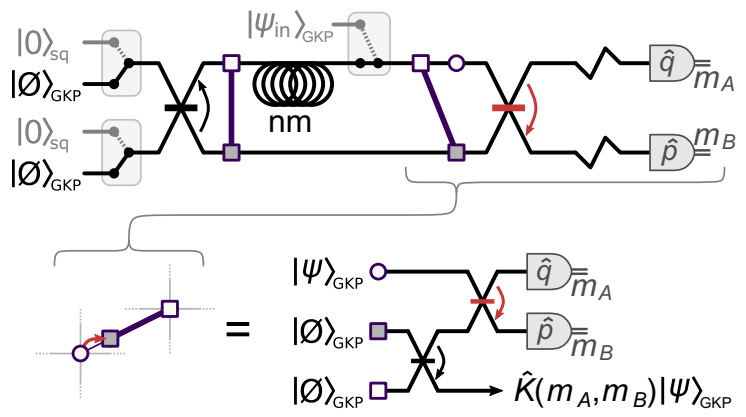

FIG. 3. Implementation of GKP quadrature correction by qubit teleportation. Qunaught states, $|\varnothing\rangle_{\mathrm{GKP}}$, are injected into the resource-preparation gadget, thereby preparing a two-mode GKP-qubit Bell state, shown as two connected rectangles before the $\mathrm{nm}$ delay. In the computational level, after the $\mathrm{nm}$ delay, one part of the Bell state overlaps in time with the GKP-qubit state to be corrected, $|\psi\rangle_{\mathrm{GKP}}$, shown as a circle. When the TDMD is set to perform a Bell measurement (the two VBSs are open and left out in the figure), $|\psi\rangle_{\mathrm{GKP}}$ is teleported through the Bell state and projected into a purified GKP qubit state by the Kraus operator in Eq. (6). At the bottom is the corresponding graph as it would appear in the 3D time lattice in Fig. 2(c), as well as the corresponding circuit diagram.

function in Eq. (4), assuming $E(q)=1$ and equal squeezing of spikes in the $\hat{q}$ and $\hat{p}$ quadratures).

The output values, $m_{A}$ and $m_{B}$, are integer multiples of $\sqrt{\pi / 2}$ plus some noise associated with the finite squeezing of the GKP qubit and qunaught states. As such, the $\hat{X}\left(-m_{A} \sqrt{2}\right)$ and $\hat{Z}\left(-m_{B} \sqrt{2}\right)$ displacements in Eq. (6) correspond mainly to Pauli- $X$ and Pauli- $Z$ operations on the encoded qubit, and are a natural result of the teleportation similar to the case of regular qubit teleportation. These displacements may be compensated for by unitarily displacing the teleported state back in $\hat{q}(\hat{p})$ by $m_{A(B)} \sqrt{2}$ rounded to the nearest integer multiple of $\sqrt{\pi}$, or simply by shifting the final measurement outcomes. However, due to the inevitable noise in $m_{A}$ and $m_{B}$, occasionally $m_{A(B)} \sqrt{2}$ is rounded to the wrong integer multiple of $\sqrt{\pi}$, which then results in a faulty displacement operation. This induces a qubit error. The probability for this error to occur is [29]

$$
p_{\sigma}(z)=\frac{\sum_{n \in \mathbb{Z}} \exp \left\{-[z-(2 n+1) \sqrt{\pi}]^{2} /\left(2 \sigma^{2}\right)\right\}}{\sum_{n \in \mathbb{Z}} \exp \left[-(z-n \sqrt{\pi})^{2} /\left(2 \sigma^{2}\right)\right]},
$$

where the residual analog information, $z=\mathcal{R}\left(m_{A(B)} \sqrt{2}\right)$, when rounding is done is given by

$$
\mathcal{R}\left(m_{A(B)} \sqrt{2}\right)=m_{A(B)} \sqrt{2}-\sqrt{\pi}\left\lfloor\frac{m_{A(B)} \sqrt{2}}{\sqrt{\pi}}+\frac{1}{2}\right\rfloor .
$$

In Eq. (7), $\sigma^{2}=\sigma_{\text {in }}^{2}+\sigma_{\mathrm{GKP}}^{2}$ is the variance of $z$, with $\sigma_{\text {in }}^{2}$ being the spike variance of the GKP qubit before teleportation. For example, if the GKP qubit to be corrected 
has gone through one gate, then $\sigma_{\text {in }}^{2}=\sigma_{\mathrm{GKP}}^{2}+\sigma_{\text {gate }}^{2}$, where $\sigma_{\mathrm{GKP}}^{2}$ was the GKP qubit spike variance before the gate and $\sigma_{\text {gate }}^{2}$ is the gate noise variance in Eq. (3). In Ref. [49], it was proposed to use the analog information from the GKP quadrature correction to improve the concatenated qubit error correction. Here, similarly to Ref. [29], we use this analog information through the probability in Eq. (7) to improve the second layer of error correction, the surface code, which is the subject of the next section.

\section{SURFACE CODE}

In Sec. III, we showed how to project the continuousvariable noise from the finite squeezing in Sec. II into qubit Pauli errors by GKP quadrature correction. However, in order to perform fault-tolerant quantum computation, such Pauli errors must then be corrected using an additional quantum error-correcting code operating at the qubit level. Given the nearest-neighbor interactions of the computation scheme in Sec. II, topological qubit error correction is a natural choice for correcting the Pauli errors. With information encoded on a surface of the computation scheme's $3 \mathrm{D}$ time lattice, and gates implemented in the third dimension, we consider a surface code [25-27]. Specifically, to compute logical $X$ or $Z$ error rates, we implement the simulation methods of Ref. [29] applied to a rotated surface code [50,51]. Such simulation methods are adapted to the computation scheme as described in Appendices B and C. We note that the rotated surface code may not be the most resource-efficient code for our computation scheme, since it is rotated by $45^{\circ}$ with respect to the $3 \mathrm{D}$ time lattice, and, thereby, computation modes located in the corner of the 3D time lattice may not be utilized - the rotated surface code is chosen in order to easily adapt the simulation method of Ref. [29]. Below, in Sec. IV A, we first describe the implementation of the surface code, and then consider it combined with GKP quadrature correction. In Sec. IV B, we then present simulation results for logical error rates and provide a squeezing threshold.

\section{A. Implementation of the rotated surface code}

A logical qubit is shown in Fig. 4(a) for a rotated surface code with distance $d=5$. Information is encoded in $d^{2}$ data qubits (white and gray circles). The stabilizers of the code are measured using $\left(d^{2}-1\right) / 2$ ancilla qubits prepared in $|+\rangle_{\mathrm{GKP}}$ (green circles) and $\left(d^{2}-1\right) / 2$ ancilla qubits prepared in $|0\rangle_{\mathrm{GKP}}$ (red circles). In what follows, we refer to the green and red ancillas as measure- $Z$ and measure- $X$ ancillas, respectively.

One round of $Z$ - and $X$-type stabilizer measurements is shown in Figs. 4(b) and 4(c). Each stabilizer measurement consists of four two-qubit gates and is thus implemented in four time steps along the third dimension of the 3D time lattice in which the surface code is implemented, as shown in Fig. 4(d). Using the optical input switch in spatial mode $A$ of the setup described in Sec. II, the ancilla qubits, initialized beforehand in the $|0\rangle_{\mathrm{GKP}}$ and $|+\rangle_{\mathrm{GKP}} \propto$ $|0\rangle_{\mathrm{GKP}}+|1\rangle_{\mathrm{GKP}}$ states, are switched into the computational level in the temporal modes corresponding to ancillary modes of the surface code. The measure- $Z$ and measure- $X$ ancillas are then coupled to neighboring data qubits using $\hat{C}_{Z}(1)=e^{i \hat{q} \otimes \hat{q}}$ and $\hat{C}_{X}( \pm 1)=e^{\mp i \hat{p} \otimes \hat{p}}$ gates, before being measured in the $\hat{p}$ and $\hat{q}$ bases, respectively. To measure such ancillas using the TDMD, the VBSs are left open, while the same basis is chosen in spatial modes $A$ and $B$, in which case the measurements commute with the beam splitter of the TDMD. Note that the state initialization and measurement bases for the ancillas are the opposite of what are traditionally used in the surface code, since they are coupled to data qubits via $\hat{C}_{Z}$ and $\hat{C}_{X}$ gates instead of sum gates, $\hat{C}_{\mathrm{NOT}}=e^{-i \hat{q} \otimes \hat{p}}$. The reason for not using sum gates is that such gates cannot be implemented in the MBQC scheme considered in this work in a single set of projective measurements. As such, using sum gates would lead to larger gate error rates compared with the error rates of the $\hat{C}_{Z}$ and $\hat{C}_{X}$ gates.

While the measure- $Z$ ancillas are coupled to data qubits with a constant coupling rate through $\hat{C}_{Z}(1)$, the measure$X$ ancillas are coupled to data qubits with $\hat{C}_{X}(1)$ in steps 1 and 4 and $\hat{C}_{X}(-1)$ in steps 2 and 3 . This is to prevent the propagation of finite squeezing noise among measure qubits [29] (though this does not matter in the case of GKP quadrature correction during the stabilizer measurements, as discussed later). Furthermore, since the $\hat{C}_{Z}(1)$ and $\hat{C}_{X}( \pm 1)$ gates cannot be implemented in a single computation step without Fourier by-products, as described in Sec. II, the surface code is implemented with the two-mode gates listed in Table I, and so, for the different two-mode gates in Fig. 4 we use

$$
\begin{aligned}
& \oint=\left\{\begin{array}{ll}
\left(\hat{F}^{\dagger} \otimes \hat{F}\right) \hat{C}_{Z}(1) & , \text { steps } 1 \text { and } 3 \\
\hat{C}_{Z}(1)\left(\hat{F} \otimes \hat{F}^{\dagger}\right) & , \text { steps } 2 \text { and } 4
\end{array},\right. \\
& \oint=\left\{\begin{array}{ll}
\left(\hat{F} \otimes \hat{F}^{\dagger}\right) \hat{C}_{X}(1) & , \text { step } 1 \\
\hat{C}_{X}(1)\left(\hat{F}^{\dagger} \otimes \hat{F}\right) & , \text { step } 4
\end{array},\right. \\
& \stackrel{O}{\vdots}=\left\{\begin{array}{ll}
\left(\hat{F} \otimes \hat{F}^{\dagger}\right) \hat{C}_{X}(-1) & \text { step 3 } \\
\hat{C}_{X}(-1)\left(\hat{F}^{\dagger} \otimes \hat{F}\right) & \text { step 2 }
\end{array}\right. \text {, }
\end{aligned}
$$

where the first term in the tensor products is the earlier temporal mode in the computational level. In this way, the Fourier by-products of steps 1 (3) and 2 (4) cancel, as $\hat{F} \hat{F}^{\dagger}=\hat{F}^{\dagger} \hat{F}=\hat{I}$ on measure- $Z$ and odd data qubits, and become $\hat{F} \hat{F}=\hat{F}^{\dagger} \hat{F}^{\dagger}=-\hat{I}$ on measure- $X$ and even data qubits. Hence, such terms have no influence on the encoded information and do not propagate errors. For CV noise, $-\hat{I}$ on even data qubits cancels with $-\hat{I}$ 


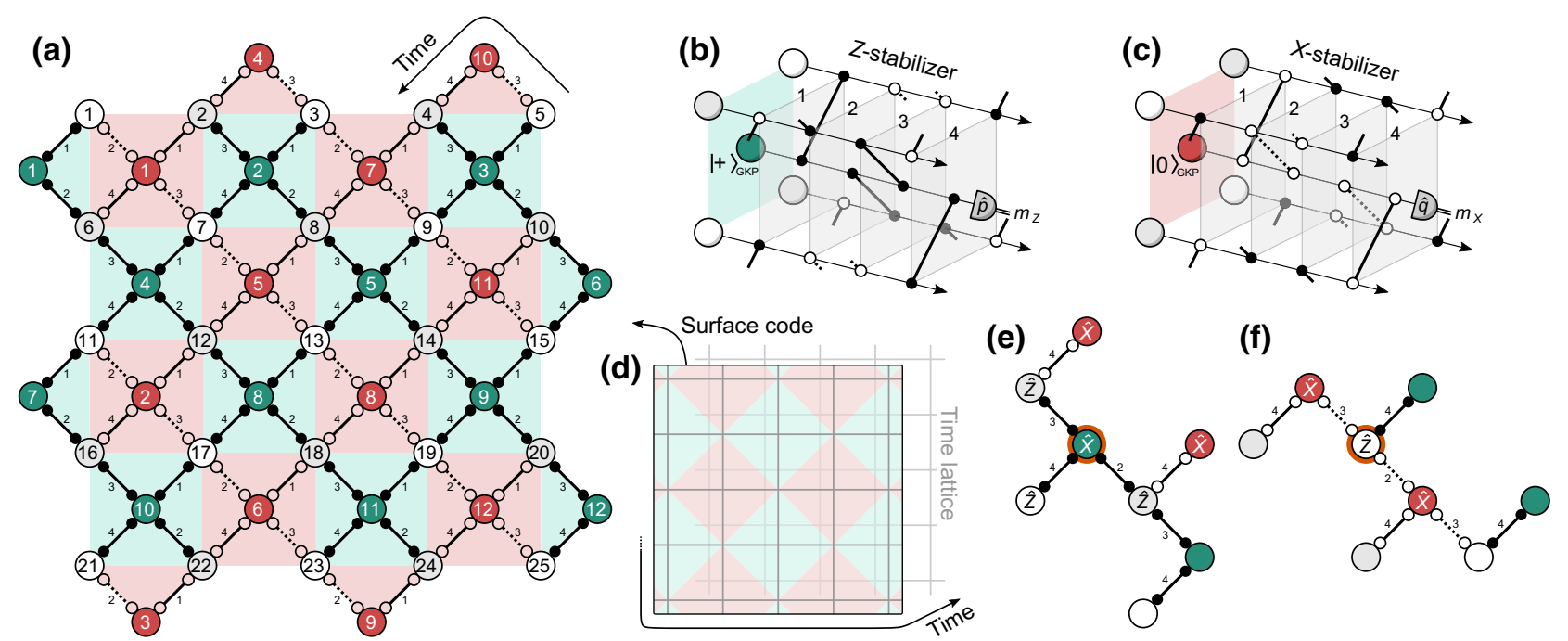

FIG. 4. (a) Illustration of a logical qubit for the $d=5$ rotated surface code. The white and gray circles represent odd and even data qubits, respectively. The green and red circles represent $Z$ - and $X$-measure qubits, respectively. The two-mode gate operations are listed in Eq. (9), and the labels 1 to 4 indicate the time steps in which those gates are implemented. (b),(c) Illustration of one round of syndrome measurements, including the initialization of the encoded GKP ancilla qubits, four time steps for the coupling between the data and ancilla qubits by four measurement-induced two-mode gates, and a measurement using the TDMD. For the surface-GKP code, GKP quadrature corrections are performed on data qubits at the beginning of a surface-code syndrome measurement cycle. For the surface-4-GKP code, GKP quadrature corrections are performed on all data and ancilla qubits after each gate. (d) Orientation of the surface code in the 3D time lattice of the computation scheme shown in Fig. 2. Here, the surface code is encoded in two dimensions of the time lattice with vertices corresponding to encoded GKP qubits, while gates are encoded in each step along the third dimension of the time lattice. (e,f) Example of qubit errors induced by GKP quadrature correction in the surface-4-GKP code. In (e), an $\hat{X}$ Pauli error occurs in a measure- $Z$ ancilla after the first two-mode gate of a surface-code syndrome measurement round. The error then propagates to neighboring data and measure- $X$ ancillas via the subsequent two-mode gates used to measure the surface-code stabilizers. In (f), a $\hat{Z}$ Pauli error occurs in a data qubit after the first two-mode gate and propagates to two measure- $X$ ancillas. See Appendix $\mathrm{C}$ for an examination of all possible Pauli errors, how they propagate, and the corresponding edges in the matching graphs for decoding.

on measure- $X$ qubits when phase-space displacements propagate between measure qubits.

We proceed to combine the surface code with GKP quadrature correction, to obtain the so-called surface-GKP code. Commonly, in the surface-GKP code, each round of syndrome measurements consists of correction of the GKP data qubits followed by measurements of the surface-code stabilizers. In this way, qubit errors induced in the GKP quadrature correction are corrected by the surface code $[18,29,52]$. However, in the usual surface-GKP code, gate noise accumulates during all four gates of the stabilizer measurements in Figs. 4(b) and 4(c). We propose to modify the scheme to perform GKP quadrature correction of each mode after every implemented gate. In other words, for each $Z$ - and $X$-stabilizer measurement, GKP quadrature correction is performed four times, and we refer to this as the surface-4-GKP code. Unfortunately, when this is done, qubit errors are induced during the surface-code stabilizer measurements, with a large impact on the fault-tolerant error threshold [25]. Two examples of induced qubit errors, and how they propagate during the stabilizer measurements, are shown in Figs. 4(e) and 4(f). Here, a qubit $\hat{X}$ error in a measure- $Z$ qubit, induced in the GKP quadrature correction after the first gate of the stabilizer measurement, propagates to three data qubits as $\hat{Z}$ errors through the $\hat{C}_{Z}$ gates (while an initial $\hat{Z}$ error will not propagate through $\hat{C}_{Z}$ ). From there, it propagates further to two measure- $X$ qubits through $\hat{C}_{X}$ gates. Similarly, a $\hat{Z}$ error in a data qubit after the first two-mode gate propagates as $\hat{X}$ errors to measure- $X$ qubits through $\hat{C}_{X}$. These errors may lead to faulty syndrome measurements and can therefore lead to wrong error recovery, inducing logic errors, but even then, we find a significant improvement of the surface-4-GKP code over the surface-GKP code. All possible Pauli errors induced by GKP quadrature correction, and their effect on the stabilizer measurements, are described in Appendix C. Note that in the case here, with GKP quadrature correction after every gate, having a coupling rate of -1 in $\hat{C}_{X}(-1)$ in steps 2 and 3 is unnecessary, as all CV noise is immediately corrected. However, since a -1 coupling rate requires no extra resources and is controlled solely by the basis settings in Table I, we keep things this way in order to compare the results with the surface-GKP code.

Finally, the surface-code $Z$ - and $X$-stabilizer measurement outcomes from $d$ rounds of syndrome measurements 
are recorded in the vertices of $3 \mathrm{D} Z$ and $X$ matching graphs with edges corresponding to possible Pauli errors, as described in Appendix C. For simplicity, here we consider only edges corresponding to single uncorrelated Pauli errors and ignore possible, but less likely, two-qubit Pauli errors correlated by two-mode gates. Minimum-weight perfect matching (MWPM) [53,54] on these matching graphs is then used as the decoding algorithm to determine data qubit errors and perform the resulting error recovery. In practice, the error recovery is handled simply by using and updating a Pauli frame [55-58], similarly to how feedforward can be handled in MBQC by compensating for by-products in the following measurement outcomes [2,3]. For the MWPM to find error paths of highest probability, the edges of the matching graphs are dynamically weighted using Eq. (7) with the residual analog information from each GKP quadrature correction. In this way, we can infer the probabilities of having induced the Pauli errors represented by each edge (described in Appendix C). With each edge representing multiple Pauli errors induced in different GKP quadrature corrections, multiple Pauli-error probabilities are combined in each edge weight as

$$
p_{\mathrm{tot}}=\frac{1}{2}\left(1-\prod_{i}\left[1-2 p_{i}\right]\right) \text {, }
$$

where $p_{i}$ is the probability given by Eq. (7) for one GKP quadrature correction, taking values between 0 (no error) and $1 / 2$ (minimal error information). For the combined probabilities of multiple edges to add up correctly in an error path determined by the MWPM, the edge weights in the matching graphs are finally taken to be $\log _{2}\left(p_{\text {tot }}\right)$.

\section{B. Simulation results}

To establish a fault-tolerant error threshold, we numerically simulate the complete scheme. The GKP-encoded data and measure qubits and the qunaught states, $|\varnothing\rangle_{\mathrm{GKP}}$, are all initialized with variance $\sigma_{\mathrm{GKP}}^{2}=\sigma^{2}=e^{-2 r} / 2$ of the wave functions' GKP spikes, as described in Sec. III. The ancillary squeezed vacuum states for gate implementation, $|0\rangle_{\mathrm{sq}}$, are also squeezed by $\sigma^{2}=e^{-2 r} / 2$, leading to quadrature-symmetric gate noise of variance $\sigma_{\text {gate }}^{2}=$ $2 \sigma^{2}=e^{-2 r}$ as described in Sec. II. Using a Monte Carlo method, logical qubit error rates are simulated as a function of squeezing using up to 100000 simulation samples, with a stopping condition at the occurrence of $500 \mathrm{com}$ bined logical $\hat{Z}$ and $\hat{X}$ qubit error events. The resulting logical $\hat{Z}$ or $\hat{X}$ error rate (the two rates are equal) is shown in Fig. 5(a) for different code distances $d$ as a function of the squeezing level, while the logical $\hat{Y}$ error rate is smaller. The decibel scale is defined relative to the vacuum variance, $10 \log _{10}\left[\sigma^{2} /(1 / 2)\right]$. The resulting squeezing threshold from where the logical error rate decreases

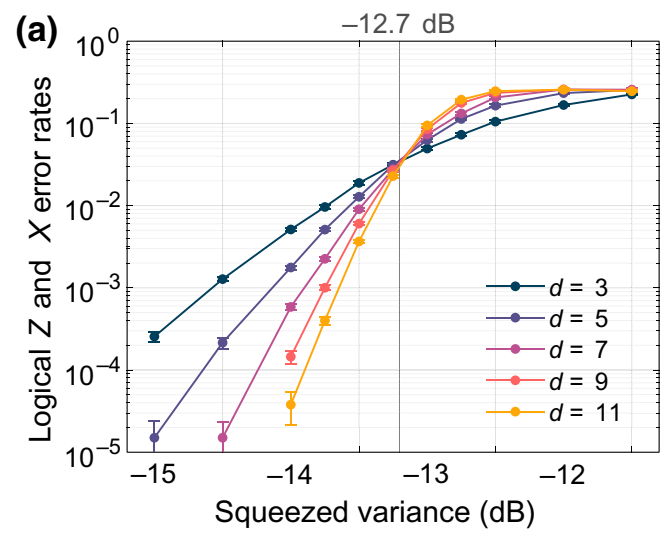

(b)

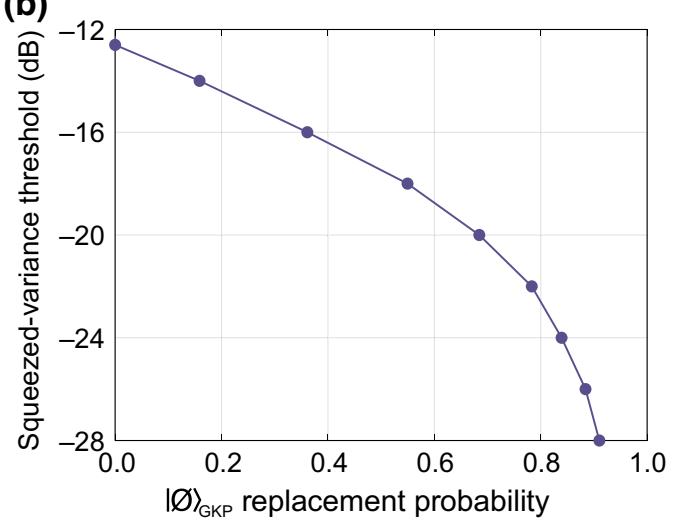

FIG. 5. (a) Simulated logical $\hat{Z}$ and $\hat{X}$ error probabilities for the surface-4-GKP code as a function of the (identical) squeezing of the $|0\rangle_{\mathrm{sq}}$ states used for gate implementation, the GKP qubits encoding the surface code, and the $|\varnothing\rangle_{\mathrm{GKP}}$ states used for quadrature correction. The logical error probability is shown for different code distances $d$, and the fault-tolerant threshold where the logical error rate decreases with increasing code distance is seen to be at $12.7 \mathrm{~dB}$ of squeezing. The error bars of standard deviation are estimated by bootstrapping. (b) Squeezing threshold of the surface-4-GKP code as a function of the probability with which a $|\varnothing\rangle_{\mathrm{GKP}}$ state is replaced with a $|0\rangle_{\mathrm{sq}}$ state in the resource preparation. Here, the threshold is estimated as the crossing point of the $d=7$ and $d=9$ logical error rates. For zero replacement probability, the threshold is that in (a).

with increasing code distance is found to be $12.7 \mathrm{~dB}$ of squeezing.

For comparison, in Appendix B we also present simulations of the error rates in other scenarios, with the simulation results shown in Fig. 6. For the surface-GKP code with a single GKP quadrature correction before the surface-code stabilizer measurements, the squeezing threshold increases to $17.3 \mathrm{~dB}$. This is significantly higher than the $12.7-\mathrm{dB}$ squeezing threshold for the surface4-GKP code due to accumulation of gate noise during the stabilizer measurements. To compare this with other MBQC schemes with topological error correction, where gate noise is typically not taken into account, and so only finite squeezing noise from GKP states is included, 
we simulate the surface-4-GKP code with $\sigma_{\text {gate }}^{2}=0$. The resulting threshold is $10.2 \mathrm{~dB}$ of squeezing, which agrees well with the $10 \mathrm{~dB}$ reported in Ref. [18] and the $10.5 \mathrm{~dB}$ reported in Ref. [21]. Finally, to see the impact of using the residual analog information about the GKP quadrature correction in the weighting of the matching graphs for MWPM decoding, we simulate the surface-4-GKP code with a fixed weighting based on the variances of each mode at each point in the code, similarly to Ref. [29]. As expected, the result is a slightly larger squeezing threshold of $13.6 \mathrm{~dB}$.

While the GKP code has been experimentally realized in trapped-ion and circuit QED systems [59-61], GKP-state generation on optical platforms is yet to be demonstrated, although there have been many recent proposals $[45,62-$ 67]. At first, GKP-state generation is most likely going to be probabilistic. In Ref. [21], it is proposed to combine multiple GKP-state generators with optical switches, and then to switch between generators with a successful preparation of a GKP state. In this way, the success probability of the generation of a GKP state, $p_{\varnothing}$, can in principle be brought arbitrarily close to 1 . Since the surface-4-GKP code requires a large supply of $|\varnothing\rangle_{\mathrm{GKP}}$ states, we consider in our final analysis the multi-GKP-state generation scheme of Ref. [21] for $|\varnothing\rangle_{\mathrm{GKP}}$ resource-state preparation. If all the generators fail to prepare a $|\varnothing\rangle_{\mathrm{GKP}}$ state in a given temporal mode for GKP correction, a deterministically generated squeezed vacuum state, $|0\rangle_{\mathrm{sq}}$, is used instead. In this case, if $|\varnothing\rangle_{\mathrm{GKP}}$ is replaced by $|0\rangle_{\mathrm{sq}}$ in spatial mode $A(B)$, only the $\hat{q}(\hat{p})$ quadrature is corrected in the GKP quadrature correction, while the other quadrature accumulates gate noise of variance $\sigma_{\text {gate }}^{2}$ during the correction [28]. The resulting fault-tolerance squeezing threshold is shown in Fig. 5(b) as a function of the probability $1-p_{\varnothing}$ of replacing $|\varnothing\rangle_{\mathrm{GKP}}$ states by $|0\rangle_{\mathrm{sq}}$ states. Here, a squeezing threshold is seen to exist over a large range of $1-p_{\varnothing}>0$, allowing a probabilistic supply of $|\varnothing\rangle_{\mathrm{GKP}}$ states, while for increasing replacement probability, the squeezing-threshold level increases as expected-above $28 \mathrm{~dB}$ of squeezing, it is hard for us to simulate the squeezing threshold due to low error rates of less than $10^{-7}$. Note that here we still assume successful encoding of the surface code. That is, the data and measure qubits switched into the setup as $\left|\psi_{\text {in }}\right\rangle_{\text {GKP }}$ in Fig. 2(a) are successfully prepared as GKP qubit states. With probabilistic optical GKP-state generation, this may be possible using state storage of a probabilistically prepared GKP state until it is switched into the computation scheme [68-71].

\section{DISCUSSION}

The squeezing thresholds in this work are derived by assuming a particular noise model in which all resource states are finitely squeezed, while all optical propagation and detection losses are set to zero. In practice, however, losses cannot be neglected. Let us denote the transmission of the setup by $\eta$. For Gaussian states, $0<\eta<1$ leads to the formation of mixed states with reduced effective squeezing. This can be reformulated as an ideal lossless setup $(\eta=1)$ with mixed squeezed vacuum states as input having a lower effective squeezing, and some excess antisqueezing that does not affect the measurement-based computation [72]. As a result, for $\eta<1$ the $12.7-\mathrm{dB}$ squeezing threshold corresponds to the effectively measured squeezing. Now, for the GKP states, besides a Gaussian convolution in the quadratures, $\eta<1$ leads to a "shrinking" of a GKP state in phase space. To see this, consider the Heisenberg picture with $\eta$ modeled as a beam splitter of transmission $\eta$. In this case, an amount $1-\eta$ of vacuum is mixed into the state, adding noise to the quadratures, while a share $1-\eta$ of the state is lost, "shrinking" the state in the quadratures by $\sqrt{\eta}$. The quadrature shrinking is more detrimental to GKP spikes far from the phase-space origin, which are naturally delimited in GKP states of finite squeezing due to the overall envelope in the quadrature wave function. For GKP states with $12.7 \mathrm{~dB}$ of squeezing, we assume the effect on the qubit error probabilities to be negligible for reasonably high efficiencies - we estimate $\eta \gtrsim 0.95$ to be doable on optical platforms. We also note that the shrinking effect can be counteracted by linear amplification, which, on the other hand, further reduces the amount of squeezing [41-44], effectively resetting $\eta$ to unity at the cost of lowering the effective squeezing and the purity of the GKP state. Again, the estimated threshold of $12.7 \mathrm{~dB}$ refers to the required squeezing after such actions have been implemented. Another detrimental effect that is not directly accounted for is interferometric phase fluctuations. Similarly to optical loss, phase fluctuations lead to mixed squeezed states of reduced squeezing and excess antisqueezing, as well as mixed GKP states, with an impact that increases with the quadrature value.

Finally, we comment on the scalability of the computation scheme. For the temporal encoding in Fig. 2, the number of modes in which GKP qubits can be encoded for computation, i.e., the size of the encoding plane in the 3D time lattice, depends on the $\mathrm{nm}$ delay in the resource-preparation gadget. Increasing the delay length increases the number of encoding modes. However, doing so also increases the optical propagation loss, which puts a limit on the useful delay length. Thus, to continue scaling up, $\mathrm{nm}$ must be increased by shortening the temporal modes, in turn increasing the demands on the squeezing and detection bandwidth. In Ref. [73], squeezed light with a bandwidth of $2.5 \mathrm{THz}$ was demonstrated, limited by the phase-matching condition for the nonlinear down-conversion process, while in Ref. [74], detection of squeezing up to a 3-THz sideband frequency was demonstrated. Assuming proper squeezing, experimental control, and detection in a $2.5-\mathrm{THz}$ bandwidth defining temporal modes of approximately $1 / 2.5 \mathrm{THz}$ duration, and assuming 
a propagation efficiency above 0.95 ( $0.23 \mathrm{~dB}$ attenuation $)$ in a low-loss optical fiber with a low optical attenuation of $0.15 \mathrm{~dB} / \mathrm{km}$, up to $n m \approx 10^{7}$ computation modes may be realized in the temporally encoded computation scheme. For the spatial architecture in Fig. 1, the scalability is similar to that of other schemes based on spatial encoding. It relies on the availability of resources, and this architecture is suitable for integrated photonics [75]. Finally, temporal and spatial encoding may be combined: Consider multiple temporally encoded computational devices, each as in Fig. 2. Using the optical switch in the computational level of the setup, computation modes can be switched in and out between different devices. Since the setup is optical, the devices are simply connected by optical fibers between the switches of each device, without the need for quantum transducers. Furthermore, with the switch being mode-selective, each mode of an encoded logical qubit in the surface code can be transferred without the need for decoding and reencoding of the logical quantum state, while measurement of the surface-code stabilizers after transfer may be used for error-correcting the transfer line. This is not only suitable for combining temporal and spatial encoding for up-scaling, but also useful in a quantum internet scheme [76,77], and is made possible by the optical architecture combined with temporal multiplexing on the transfer lines.

\section{CONCLUSION}

In this work, we propose a simple but complete and scalable architecture for optical CV MBQC that includes quadrature noise correction and qubit error correction using topological codes. The setup consists of simple optical devices such as beam splitters, delays, optical switches, and variable beam splitters, where the latter two can be decomposed into beam splitters and optical phase shifters. The scheme allows both spatial and temporal encoding, with the temporally encoded version requiring just two squeezing sources. A universal Gaussian gate set is directly implementable, while universal qubit computation is made possible by feeding the setup with GKP states, thereby supplying the required non-Gaussianity [78-80]. As the computation scheme is based on gate teleportation on wires of two-mode entangled states, the setup naturally supports the new GKP quadrature correction scheme presented in Ref. [28], circumventing the need for online two-mode gates coupled to ancillary GKP states. Finally, by arranging the GKP qubits in a 2D plane of the cluster state that allows nearest-neighbor interactions, topological codes can be realized. By encoding a variation of the surface-GKP code - the surface-4-GKP code-we show fault-tolerant computation to be possible above a certain squeezing threshold by simulating a logical qubit memory, or an identity gate, of the surface-4-GKP code. In the surface code, Clifford gates can be implemented by braiding
[25] or lattice surgery [81], which is implemented by regulating the surface-code syndrome measurements, while non-Clifford gates may be realized using magic states distilled from GKP qubits prepared in a magic state $[78,79]$ and injected into the surface code as input states [25,82].

The fault-tolerant squeezing threshold is found to be $12.7 \mathrm{~dB}$. The estimation of this number takes into account the finite squeezing values of GKP states, as well as the gate noise stemming from the finite squeezing values of the generated cluster state on which gates are implemented by projective measurements. However, this squeezing threshold leaves room for improvement: In the matching graph of the decoding algorithm, we consider only single uncorrelated Pauli errors. As an improvement, assuming the surface code to consist of two-mode gates, we may consider a matching graph taking two-qubit error events into account [83-86]. Furthermore, when weighting the matching-graph edges with analog information from the GKP quadrature correction, we simply consider uncorrelated noise in the individual GKP corrections. However, since each mode is subjected to a two-mode gate prior to GKP correction, leading to correlated noise on neighboring GKP qubits, we may improve the estimation of the Pauli-error probabilities used in the matching-graph weighting by jointly considering the analog information from neighboring GKP corrections, as recently proposed in Ref. [87]. Note that such modifications are implemented solely at the software level of the error-correction decoder and thus require no modifications to the setup. Another improvement may be found in the gate implementation: Because of the similarity of the GKP quadrature correction and the gate implementation, it might be possible to combine the two transformations into one step, that is, to implement a gate while correcting the quadratures. Although the quadrature correction is considered only on single wires [28], it might be possible to generalize it to the two-wire case, whereby two-mode gates could be implemented during GKP quadrature correction, thereby eliminating the gate noise caused by finite squeezing. If this is possible while maintaining the quality of the GKP quadrature correction in Ref. [28], the resulting squeezing threshold reduces to $10.2 \mathrm{~dB}$, as shown in Appendix B.

\section{ACKNOWLEDGMENTS}

The work was supported by the Danish National Research Foundation through the Center for Macroscopic Quantum States (bigQ, DNRF0142).

\section{APPENDIX A: GATES BY PROJECTIVE MEASUREMENTS}

To derive the gates implemented by projective measurements in Sec. II, consider one computation step on two 
parallel wires,

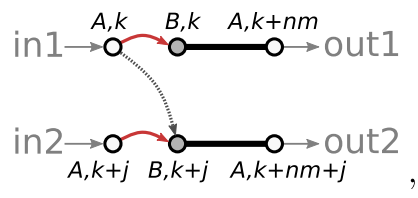

where the red arrows represent the first beam splitter in the TDMD, and the gray arrow represents the blue or green VBS in the TDMD for $j=1$ or $j=n$, respectively. Here, a two-mode input state (which may or may not be separable) is encoded in modes $(A, k),(A, k+j)$, while the two-mode entangled states are prepared in the resource-preparation gadget, and can be written as

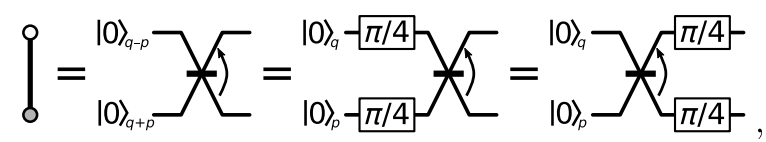

where $|0\rangle_{q-p}$ and $|0\rangle_{q+p}$ are squeezed vacuum states, squeezed along the $(\hat{q}-\hat{p}) / \sqrt{2}$ and $(\hat{q}+\hat{p}) / \sqrt{2}$ quadratures, respectively, and, similarly, $|0\rangle_{q}$ and $|0\rangle_{p}$ are squeezed along $\hat{q}$ and $\hat{p}$, respectively. As such, the twomode entangled states correspond to two-mode squeezed states rotated in phase space by $\pi / 4$, turning them into approximate cluster states with edge weight $\tanh 2 r$ and self-loops $i \operatorname{sech} 2 r$, where $r$ is the squeezing parameter of the initial squeezed vacuum states [19,33]. Alternatively, we can consider the two-mode entangled states more generally as cluster-type states [32], here with edge weight 1 , for which the implemented gate is independent of the squeezing $r$, which then affects only the gate noise [10]. The two situations are equivalent: one can change from the former to the latter by normalizing the edge weight [30]. Here, we consider cluster-type states, since implementing a desired gate in practice (without considering the resulting gate noise) then requires no prior knowledge of the squeezing level.

For single-mode gates, the VBSs of the TDMD are left open, and the dashed arrow in Eq. (A1) represents $\hat{I}_{A, k} \otimes \hat{I}_{B, k+j}$. In this case, we can ignore the second wire, and focus on a joint projective measurement of the input mode $(A, k)$ and one mode of the two-mode entangled state, $(B, k)$, resulting in gate teleportation to the output mode $(A, k+n m)$ - exactly the same derivation can be done for the second wire for modes $(A, k+j),(B, k+j)$, and $(B, k+n m+j)$. The corresponding circuit is

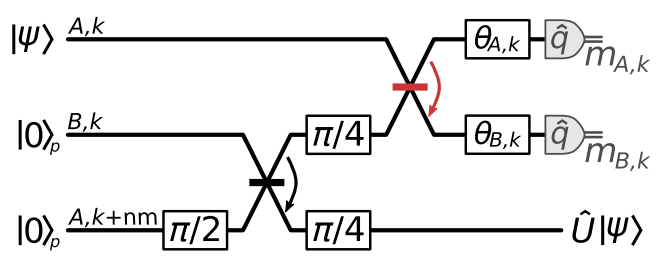

where $|0\rangle_{q}$ in $(A, k+n m)$ is replaced by $\hat{R}(\pi / 2)|0\rangle_{p}$ only to follow the traditional convention of using cluster states with an initial squeezing in the $\hat{p}$ quadratures. Using the method of Appendix A of Ref. [31], with $\left(\theta_{A, k}, \theta_{B, k}\right)$ being the basis setting determining the implemented gate, the corresponding quadrature transformation in the Heisenberg picture can be derived as

$$
\left(\begin{array}{c}
\hat{q}_{A, k+n m}^{\prime} \\
\hat{p}_{A, k+n m}^{\prime}
\end{array}\right)=\mathbf{G}\left(\begin{array}{c}
\hat{q}_{A, k} \\
\hat{p}_{A, k}
\end{array}\right)+\mathbf{N}\left(\begin{array}{c}
\hat{p}_{B, k} \\
\hat{p}_{A, k+n m}
\end{array}\right)+\mathbf{D}\left(\begin{array}{c}
m_{A, k} \\
m_{B, k}
\end{array}\right) .
$$

Here, $\mathbf{G}$ is the symplectic matrix corresponding to the desired single-mode gate operation in Eq. (2),

$$
\mathbf{N}=\left(\begin{array}{cc}
1 & 1 \\
1 & -1
\end{array}\right)
$$

is a gate-noise matrix, and

$$
\mathbf{D}=\frac{\sqrt{2}}{\sin \left(2 \theta_{-}\right)}\left(\begin{array}{cc}
-\cos \theta_{B, k} & -\cos \theta_{A, k} \\
\sin \theta_{B, k} & \sin \theta_{A, k}
\end{array}\right)
$$

is a displacement matrix. Since $\left(\theta_{A, k}, \theta_{B, k}\right), m_{A, k}$, and $m_{B, k}$ are known, $\mathbf{D}\left(m_{A, k}, m_{B, k}\right)^{T}$ can be compensated for by displacing the teleported state back by $-\mathbf{D}\left(m_{A, k}, m_{B, k}\right)^{T}$, or simply by taking this displacement into account in the following measurement outcomes. With finite squeezing in the ancillary modes such that $\operatorname{Var}\left\{\hat{p}_{B, k}\right\}=\operatorname{Var}\left\{\hat{p}_{A, k+n m}\right\}=$ $\sigma^{2}=e^{-2 r} / 2$, the noise term $\mathbf{N}\left(\hat{p}_{B, k}, \hat{p}_{A, k+n m}\right)^{T}$ leads to quadrature-symmetric gate noise in $\hat{q}_{A, k+n m}^{\prime}$ and $\hat{p}_{A, k+n m}^{\prime}$ of

$$
\sigma_{\text {gate }}^{2}=\operatorname{Var}\left\{\hat{p}_{B, k}\right\}+\operatorname{Var}\left\{\hat{p}_{A, k+n m}\right\}=e^{-2 r} .
$$

In the Wigner-function picture, this gate noise corresponds to convolutions in both quadratures by a Gaussian function of variance $\sigma_{\text {gate }}^{2}$, each followed by the application of a corresponding Gaussian envelope due to the Fourier relation between $\hat{q}$ and $\hat{p}[30,31]$.

When two-mode gates are implemented by enabling the first or second VBS of the TDMD, the corresponding circuit is

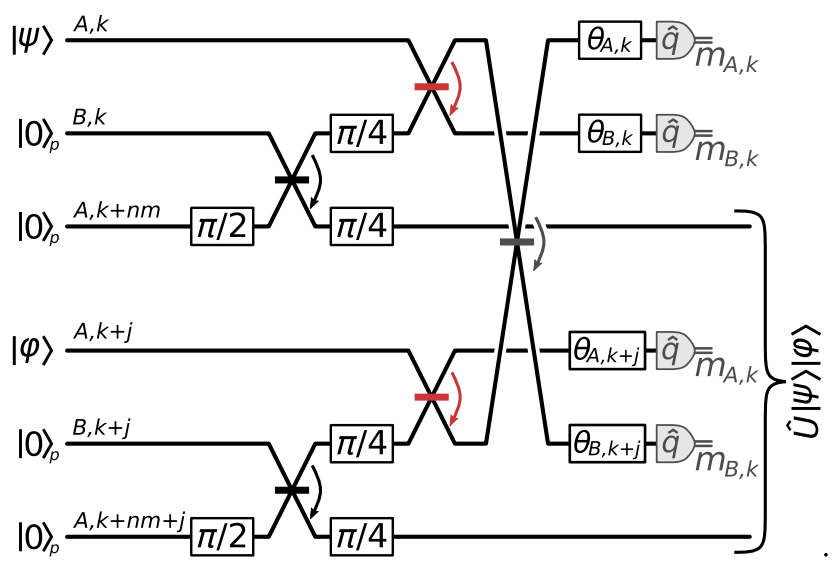


We do not derive a general expression for the implemented gate as a function of the basis setting $\left(\theta_{A, k}, \theta_{B, k}, \theta_{A, k+j}, \theta_{B, k+j}\right)$. Instead, we use the method described in Ref. [31]: a cost function is defined based on the implemented gate, a desired target gate, and the gate noise, which is then used in a global search to find the basis setting that implements a desired gate with the minimum gate noise. The resulting basis settings for the gates required to implement the surface code are shown in Table I. We note that those settings implement only two different gates, since $\left(\hat{F}^{\dagger} \otimes \hat{F}\right) \hat{C}_{Z}(g)=\hat{C}_{X}(g)\left(\hat{F}^{\dagger} \otimes\right.$ $\hat{F})$ and $\hat{C}_{Z}(g)\left(\hat{F} \otimes \hat{F}^{\dagger}\right)=\left(\hat{F} \otimes \hat{F}^{\dagger}\right) \hat{C}_{X}(g)$. The reason for considering them as four different gates is to make the implementation of the surface code more intuitive. The basis settings are not unique: other settings exist that implement the same gates with equal gate noise.

The quadrature transformation when the basis settings for two-mode gates are applied is

$$
\begin{aligned}
\left(\begin{array}{c}
\hat{q}_{A, k+n m}^{\prime} \\
\hat{q}_{A, k+n m+j}^{\prime} \\
\hat{p}_{A, k+n m}^{\prime} \\
\hat{p}_{A, k+n m+j}^{\prime}
\end{array}\right)= & \mathbf{G}\left(\begin{array}{c}
\hat{q}_{A, k} \\
\hat{q}_{A, k+j} \\
\hat{p}_{A, k} \\
\hat{p}_{A, k+j}
\end{array}\right)+\mathbf{N}\left(\begin{array}{c}
\hat{p}_{B, k} \\
\hat{p}_{A, k+n m} \\
\hat{p}_{B, k+j} \\
\hat{p}_{A, k+n m+j}
\end{array}\right) \\
& +\mathbf{D}\left(\begin{array}{c}
m_{A, k} \\
m_{B, k} \\
m_{A, k+j} \\
m_{B, k+j}
\end{array}\right) .
\end{aligned}
$$

Again, $\mathbf{G}$ is the symplectic matrix corresponding to the implemented two-mode gate. $\mathbf{D}\left(m_{A, k}, m_{B, k}, m_{A, k+j}, m_{B, k+j}\right)^{T}$ is a displacement in phase space, with

$$
\mathbf{D}=\left(\begin{array}{cccc}
-\sqrt{5} / 2 & 0 & 1 / \sqrt{2} & \sqrt{5} / 2 \\
-\sqrt{5} / 2 & -1 / \sqrt{2} & 0 & -\sqrt{5} / 2 \\
0 & -\sqrt{2} & 0 & 0 \\
0 & 0 & \sqrt{2} & 0
\end{array}\right)
$$

for $\left(\hat{F}^{\dagger} \otimes \hat{F}\right) \hat{C}_{Z}(g)$ and $\hat{C}_{X}(g)\left(\hat{F}^{\dagger} \otimes \hat{F}\right)$, and

$$
\mathbf{D}=\left(\begin{array}{cccc}
0 & -\sqrt{2} & 0 & 0 \\
0 & 0 & -\sqrt{2} & 0 \\
\sqrt{5} / 2 & 0 & -1 / \sqrt{2} & \sqrt{5} / 2 \\
\sqrt{5} / 2 & -1 / \sqrt{2} & 0 & -\sqrt{5} / 2
\end{array}\right)
$$

for $\hat{C}_{Z}(g)\left(\hat{F} \otimes \hat{F}^{\dagger}\right)$ and $\left(\hat{F} \otimes \hat{F}^{\dagger}\right) \hat{C}_{X}(g)$, both of which can be compensated for, just as for single-mode gates. $\mathbf{N}\left(\hat{p}_{B, k}, \hat{p}_{A, k+n m}, \hat{p}_{B, k+j}, \hat{p}_{A, k+n m+j}\right)^{T}$ represents gate noise, where

$$
\mathbf{N}=\left(\begin{array}{cccc}
1 & 1 & 0 & 0 \\
0 & 0 & 1 & 1 \\
1 & -1 & 0 & 0 \\
0 & 0 & 1 & -1
\end{array}\right)
$$

leads to quadrature-symmetric gate noise of variance

$$
\begin{aligned}
\sigma_{\text {gate }}^{2} & =\operatorname{Var}\left\{\hat{p}_{B, k}\right\}+\operatorname{Var}\left\{\hat{p}_{A, k+n m}\right\} \\
& =\operatorname{Var}\left\{\hat{p}_{B, k+j}\right\}+\operatorname{Var}\left\{\hat{p}_{A, k+n m+j}\right\} \\
& =e^{-2 r}
\end{aligned}
$$

which conveniently equals the gate-noise variance of single-mode gates.

\section{APPENDIX B: SIMULATION}

To simulate the logical qubit error rate of the surface code, we adopt and modify the simulation presented in Ref. [29] for the computation scheme of this work. The simulation method is well described in Appendix B of Ref. [29] and is summarized here with focus on the modifications. In the simulation, quadrature noise is simulated as stochastic normally distributed variables for each quadrature of each mode $i, \xi_{q}^{i}$ and $\xi_{p}^{i}$. For GKP states, $\xi_{q}^{i}$ and $\xi_{p}^{i}$ are initialized with random samples from $\mathcal{N}\left(0, \sigma_{\mathrm{GKP}}\right)$, where $\mathcal{N}(0, \sigma)$ is a normal distribution with zero mean and variance $\sigma^{2}$. After each gate, independent random samples from $\mathcal{N}\left(0, \sigma_{\text {gate }}\right)$ are added to $\xi_{q}^{i}$ and $\xi_{p}^{i}$ as gate noise. For homodyne measurements, $\xi_{q}^{i}$ or $\xi_{p}^{i}$ is read out, and the logic value is determined from the closest integer multiple of $\sqrt{\pi}$. Note that, unlike in Ref. [29], we do not consider measure noise or idle noise. On optical platforms, homodyne measurements are carried out with near-unity efficiency (any loss is assumed to just degrade the squeezing, as discussed in Sec. V). Furthermore, in MBQC no modes are idle, since modes not performing any tasks still have to be teleported through the computation step, and thereby acquire gate noise instead of idle noise.

For the two-mode gates in the surface code, the simulation here differs from that in Ref. [29] by using $\hat{C}_{Z}(1)$ and $\hat{C}_{X}( \pm 1)$ gates instead of sum gates. For a two-mode gate between modes $i$ and $j$, the quadrature noise variables are updated as follows:

$$
\begin{aligned}
\xi_{q}^{i} \leftarrow \xi_{q}^{i}+\operatorname{randG}\left(\sigma_{\text {gate }}^{2}\right), \\
\xi_{q}^{i} \leftarrow \xi_{p}^{i}+\xi_{q}^{j}+\operatorname{randG}\left(\sigma_{\text {gate }}^{2}\right), \\
\hat{C}_{Z}(1): \xi_{q}^{j}+\operatorname{randG}\left(\sigma_{\text {gate }}^{2}\right), \\
\xi_{q}^{j} \leftarrow \xi_{p}^{j}+\xi_{q}^{i}+\operatorname{randG}\left(\sigma_{\text {gate }}^{2}\right), \\
\xi_{q}^{i} \leftarrow \xi_{q}^{i} \pm \xi_{p}^{j}+\operatorname{randG}\left(\sigma_{\text {gate }}^{2}\right), \\
\xi_{q}^{i} \leftarrow \xi_{p}^{i}+\operatorname{randG}\left(\sigma_{\text {gate }}^{2}\right), \\
\hat{C}_{X}( \pm 1): \leftarrow \xi_{q}^{j} \pm \xi_{p}^{i}+\operatorname{randG}\left(\sigma_{\text {gate }}^{2}\right), \\
\xi_{q}^{j} \leftarrow \xi_{p}^{j}+\operatorname{randG}\left(\sigma_{\text {gate }}^{2}\right),
\end{aligned}
$$

where randG $\left(\sigma^{2}\right)$ returns a random value from $\mathcal{N}(0, \sigma)$.

For GKP quadrature correction, instead of being coupled to ancillary GKP qubits through sum gates as in Ref. [29], the mode to be corrected is teleported through 
a two-mode GKP qubit Bell state as described in Sec. III. The Bell state is prepared by interfering two GKP qunaught states, denoted by $\varnothing 1$ and $\varnothing 2$, where

$$
\begin{array}{ll}
\xi_{q}^{\varnothing 1}=\operatorname{randG}\left(\sigma_{\mathrm{GKP}}^{2}\right), & \xi_{q}^{\varnothing 2}=\operatorname{randG}\left(\sigma_{\mathrm{GKP}}^{2}\right), \\
\xi_{p}^{\varnothing 1}=\operatorname{randG}\left(\sigma_{\mathrm{GKP}}^{2}\right), & \xi_{p}^{\varnothing 2}=\operatorname{randG}\left(\sigma_{\mathrm{GKP}}^{2}\right),
\end{array}
$$

on a beam splitter:

$$
\begin{aligned}
& \xi_{q}^{\varnothing 1} \leftarrow\left(\xi_{q}^{\varnothing 1}-\xi_{q}^{\varnothing 2}\right) / \sqrt{2}, \\
& \xi_{p}^{\varnothing 1} \leftarrow\left(\xi_{p}^{\varnothing 1}-\xi_{p}^{\varnothing 2}\right) / \sqrt{2}, \\
& \xi_{q}^{\varnothing 2} \leftarrow\left(\xi_{q}^{\varnothing 1}+\xi_{q}^{\varnothing 2}\right) / \sqrt{2}, \\
& \xi_{p}^{\varnothing 2} \leftarrow\left(\xi_{p}^{\varnothing 1}+\xi_{p}^{\varnothing 2}\right) / \sqrt{2} .
\end{aligned}
$$

To perform the teleportation, the mode to be corrected, $i$, and $\varnothing 1$ are interfered on a beam splitter and measured in $\hat{q}$ and $\hat{p}$, respectively, with outcomes

$$
m_{A}=\left(\xi_{q}^{i}-\xi_{q}^{\varnothing 1}\right) / \sqrt{2}, \quad m_{B}=\left(\xi_{p}^{i}+\xi_{p}^{\varnothing 1}\right) / \sqrt{2} .
$$

Finally, to compensate for the Pauli by-products of the qubit teleportation (displacements by $\sqrt{\pi}$ ), $m_{A} \sqrt{2}$ and $m_{B} \sqrt{2}$ are rounded to the nearest integer multiple of $\sqrt{\pi}$, i.e.,

$$
\mathcal{P}\left(m_{A(B)} \sqrt{2}\right)=\sqrt{\pi}\left\lfloor\frac{m_{A(B)} \sqrt{2}}{\sqrt{\pi}}+\frac{1}{2}\right\rfloor,
$$

which is then used to displace the teleportation output mode, $\varnothing 2$, back:

$$
\begin{aligned}
& \xi_{q}^{\varnothing 2} \leftarrow \xi_{q}^{\varnothing 2}+\mathcal{P}\left(m_{A} \sqrt{2}\right), \\
& \xi_{p}^{\varnothing 2} \leftarrow \xi_{p}^{\varnothing 2}+\mathcal{P}\left(m_{B} \sqrt{2}\right) .
\end{aligned}
$$

For the sake of simulation, we pass the corrected output mode to the input mode, i.e., $\xi_{q}^{i} \leftarrow \xi_{q}^{\varnothing 2}$ and $\xi_{p}^{i} \leftarrow \xi_{p}^{\varnothing 2}$, such that mode $i$ can be reused in the following simulation. The probability of having induced a qubit error by rounding to a wrong integer multiple of $\sqrt{\pi}$ in Eq. (B1) due to input noise in $\xi_{q}^{i}$ and $\xi_{p}^{i}$, together with initialization noise in $\xi_{q}^{\varnothing 1}, \xi_{p}^{\varnothing 1}, \xi_{q}^{\varnothing 2}$, and $\xi_{p}^{\varnothing 2}$, is inferred using the residual ana$\log$ information, $\mathcal{R}\left(m_{A(B)} \sqrt{2}\right)=m_{A(B)} \sqrt{2}-\mathcal{P}\left(m_{A(B)} \sqrt{2}\right)$, in Eq. (8) through the probability in Eq. (7). Finally, this probability is used for weighting the matching graphs of stabilizer measurement outcomes for the MWPM decoding as described in Appendix C. Here, $\sigma_{\text {in }}^{2}$ in $\sigma^{2}=\sigma_{\text {in }}^{2}+\sigma_{\mathrm{GKP}}^{2}$ of Eq. (7) is the quadrature variance of the input mode, and is carefully kept track of in the simulation based on previous gates and corrections.

In one simulation, $d+1$ rounds of surface-code stabilizer measurements are carried out. Data GKP qubits are initialized in round 1 with variance $\sigma_{\mathrm{GKP}}^{2}$. To stabilize the data qubits, measure GKP qubits and qunaught states are initialized in rounds 1 to $d$ with variance $\sigma_{\mathrm{GKP}}^{2}$, followed by noisy gates and measurements to build up the matching graphs. In the last round, $d+1$, measure GKP qubits and qunaught states are initialized with zero variance to carry out ideal syndrome measurements for determining the logical qubit errors induced in rounds 1 to $d$. To build up statistics, for each squeezing level and code distance $d$, this process is repeated 100000 times or until a total of 500 logical $\hat{X}$ and $\hat{Z}$ errors are detected.

We simulate four different cases, shown in Fig. 6. In three cases, GKP states (qubits and qunaught states) and squeezed vacuum states are initialized with equal variance, $\sigma_{\mathrm{GKP}}^{2}=\sigma^{2}=e^{-2 r} / 2$, which from Eqs. (3) and (5) leads to $\sigma_{\text {gate }}^{2}=2 \sigma_{\mathrm{GKP}}^{2}$. In this way, the surface-GKP code with GKP quadrature correction before the surface-code stabilizer measurements and the surface-4-GKP code with

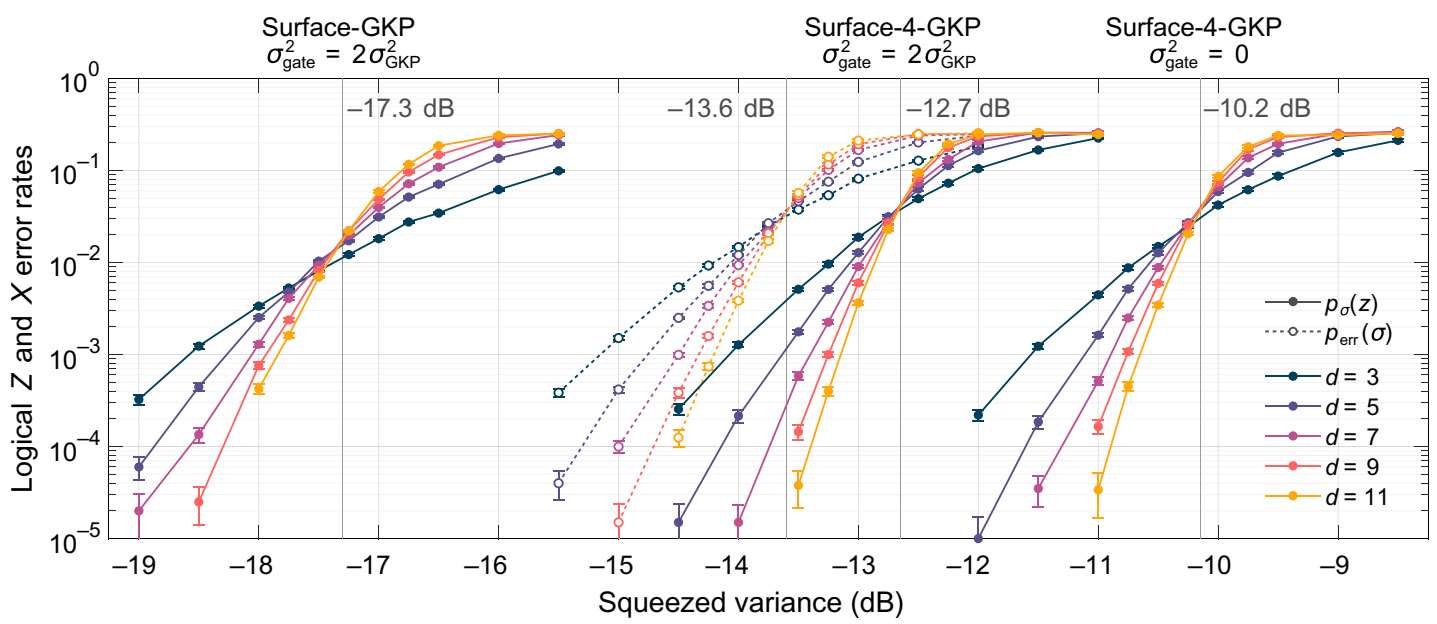

FIG. 6. Simulation results for all four simulated cases. Here, the case of the surface-4-GKP code using $p_{\sigma}(z)$ gives the results shown in the main text in Fig. 5. The error bars of standard deviation are estimated by bootstrapping. 
four GKP quadrature corrections during the stabilizer measurements are simulated. To see the impact of using the analog information from the GKP correction in the weighting of the matching graphs, the surface-4-GKP code is simulated using

$$
p_{\mathrm{err}}(\sigma)=\sum_{n \in \mathbb{Z}} \frac{1}{\sqrt{2 \pi \sigma^{2}}} \int_{(2 n+1 / 2) \sqrt{\pi}}^{(2 n+3 / 2) \sqrt{\pi}} d \xi e^{-\xi^{2} /\left(2 \sigma^{2}\right)}
$$

instead of Eq. (7) [29]. By integrating the wave-function marginal distribution in the odd GKP bins, $p_{\text {err }}(\sigma)$ infers the qubit error probability based only on variances, without taking the projective-measurement outcome into account. Finally, to compare the results with other MBQC schemes supporting topological error correction, but taking only noise from GKP states into account, the surface-4-GKP code is simulated using $\sigma_{\text {gate }}^{2}=0$.

\section{APPENDIX C: DECODING GRAPHS}

In this appendix, we describe the matching graphs used in the MWPM decoding. Sections of the $Z$ and $X$ matching graphs are shown in Fig. 7. Each vertex corresponds to a syndrome measurement and is highlighted when the measurement outcome changes relative to the previous measurement of the same measure qubit, indicating an error event. The edges correspond to possible errors, and the job of the decoder is to match pairs of highlighted vertices with the most likely error path, which is then translated into corrections of data qubits.

Each horizontal plane of the matching graphs in Fig. 7 corresponds to one round, $i$, of the syndrome measurements in Figs. 4(b) and 4(c). We distinguish between four different types of edge: horizontal edges, $h$; vertical edges, $v$; diagonal edges, $d$; and cross edges, $c$. In the case where errors occur only in between syndrome measurements [i.e., before and after the syndrome-measurement circuits in Figs. 4(b) and 4(c)], errors in data qubits correspond to $h$ edges, while errors in the syndrome-measurement readout correspond to $v$ edges. These are often the only edges included in the surface-code matching graphs when errors during the syndrome measurements are not considered. However, for the surface-4-GKP code with GKP quadrature correction during the syndrome measurements, most qubit errors are induced during the syndrome measurements, requiring the additional $d$ and $c$ edges (often referred to as space-time edges) in the matching graphs for optimal decoding [83].

Below, we go through all possible qubit errors that may be induced by each GKP quadrature correction in each of the four steps in the syndrome measurements, and describe the corresponding edge in the matching graphs. For simplicity, in this work we consider only uncorrelated single-qubit errors. Since the surface code consists of twomode gates, two-qubit errors are possible as well, but we
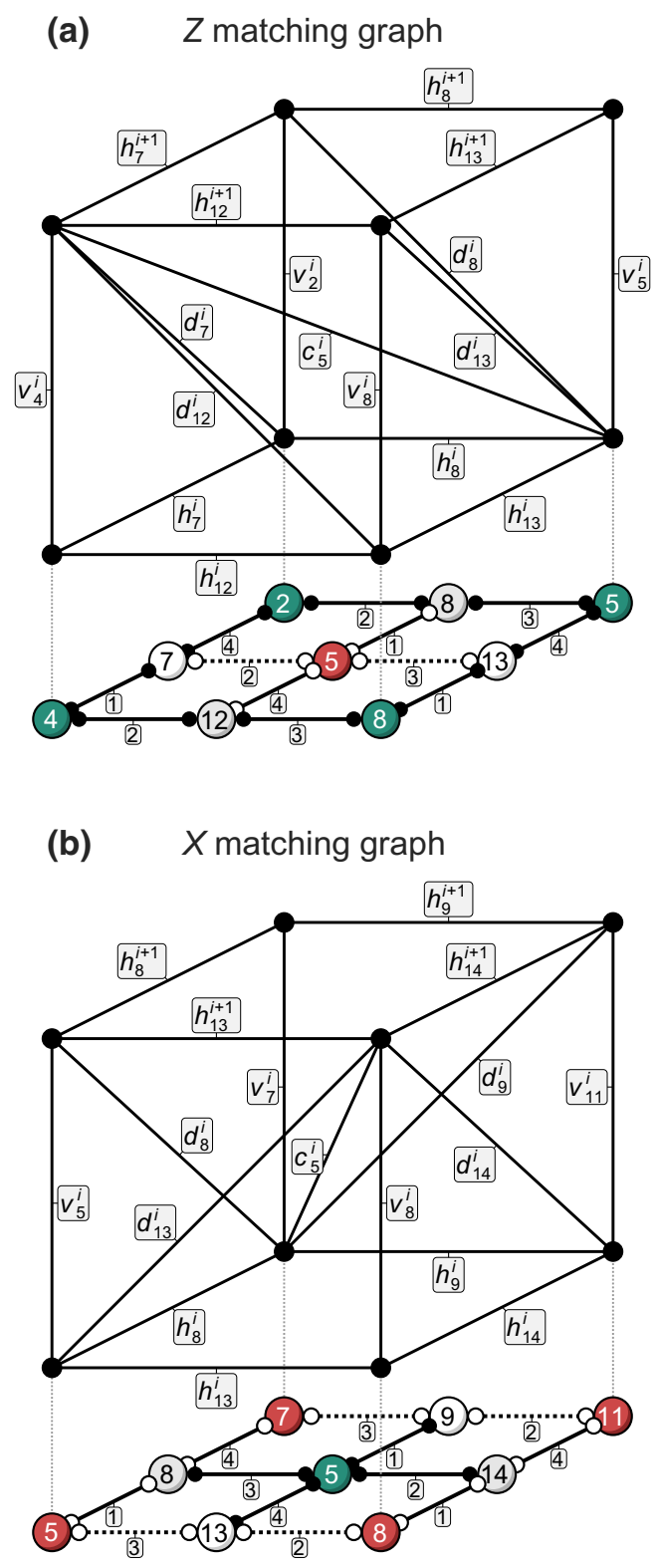

FIG. 7. Sections of the $Z$ (a) and $X$ (b) matching graphs, here with labels corresponding to the code distance $d=5$ similarly to Fig. 4. Each two-mode gate is labeled with a number corresponding to the step in the $i$ th round of syndrome measurements.

assume them to be negligible in the squeezing range considered. The effect of two-qubit errors on the matching graphs is shown in Ref. [83]. Finally, in Sec. 5, we comment on the graph boundaries, edge weighting, and qubit correction.

\section{Step 1}

After the first set of two-mode gates in the syndrome measurements (step 1), we perform GKP quadrature correction. At this point, we consider single-qubit Pauli- $X$ 
errors, $\hat{X}=e^{ \pm i \sqrt{\pi} \hat{p}}$, and Pauli- $Z$ errors, $\hat{Z}=e^{ \pm i \sqrt{\pi} \hat{q}}$ (corresponding to displacements of $\sqrt{\pi}$ in $\hat{q}$ and $\hat{p}$, respectively), in each data and measure qubit. In all the following, we refer to the $j$ th data, measure- $Z$, and measure- $X$ qubits as $D_{j}, Z_{j}$, and $X_{j}$, respectively, and we use the labeling in Fig. 7 for the code with distance $d=5$ as an example.

For an odd data qubit, say $D_{13}$, an $\hat{X}$ error is detected by $Z_{5}$ in step 4 of the current round, $i$, while it is detected by $Z_{8}$ in step 1 of the following round, $i+1$. As a result, this error corresponds to the edge $d_{13}^{i}$ of the $Z$ graph. A $\hat{Z}$ error is detected in $X_{8}$ and $X_{5}$ in steps 2 and 3 of the current round $i$ and corresponds to $h_{13}^{i}$ in the $X$ graph.

For an even data qubit, say $D_{8}$, an $\hat{X}$ error is detected by $Z_{2}$ and $Z_{5}$ in steps 2 and 3 of the current round, $i$, and corresponds to $h_{8}^{i}$ in the $Z$ graph. A $\hat{Z}$ error is detected by $X_{7}$ in step 4 of the current round, $i$, and by $X_{5}$ in step 1 of the following round, $i+1$. Thus this error corresponds to $d_{8}^{i}$ in the $X$ graph.

For the measure- $Z$ qubit $Z_{5}$, an $\hat{X}$ error propagates to $D_{8,13,14}$ through $\hat{C}_{Z}(1)$ in steps 2,3 , and 4 , where it causes a $\hat{Z}$ error, which eventually highlights $X_{7}$ and $X_{11}$ in step 4 of this round $i$, indicating a false error in $D_{9}$ instead of in $D_{8,13,14}$. However, applying a $\hat{Z}$ correction to $D_{9}$ constitutes, together with the $\hat{Z}$ errors in $D_{8,13,14}$, a code stabilizer, and the error is successfully corrected. Thus, an $\hat{X}$ error in $Z_{5}$ corresponds to $h_{9}^{i}$ in the $X$ graph. A $\hat{Z}$ error in $Z_{5}$ does not propagate through the $\hat{C}_{Z}(1)$ gates, and becomes instead a detection error at the end of the syndrome measurement, corresponding to $v_{5}^{i}$ in the $Z$ graph.

For the measure- $X$ qubit $X_{5}$, an $\hat{X}$ error does not propagate through the $\hat{C}_{X}( \pm 1)$ gates, and becomes a detection error at the end of the syndrome measurement, corresponding to $v_{5}^{i}$ in the $X$ graph. A $\hat{Z}$ error propagates as $\hat{X}$ errors to $D_{7,12,13}$ through $\hat{C}_{X}( \pm 1)$ gates, which eventually highlights $Z_{2}$ and $Z_{5}$ in step 4 of this round, indicating a false error in $D_{8}$. Applying an $\hat{X}$ correction to $D_{8}$ constitutes, together with the $\hat{X}$ errors in $D_{7,12,13}$, a code stabilizer, and the error is successfully corrected. Thus a $\hat{Z}$ error in $X_{5}$ corresponds to $h_{8}^{i}$ in the $Z$ graph.

\section{Step 2}

Below, we consider single-qubit errors induced in the GKP quadrature correction after the second set of twomode gates in the syndrome measurements (step 2).

An $\hat{X}$ error in an odd data qubit is detected similarly to an $\hat{X}$ error induced in step 1 . A $\hat{Z}$ error in an odd data qubit, say $D_{13}$, is detected by $X_{5}$ in step 3 of this round, $i$, while it is detected by $X_{8}$ in step 2 of round $i+1$. Thus the corresponding edge is $d_{13}^{i}$ in the $X$ graph.

An $\hat{X}$ error in an even data qubit, say $D_{8}$, is detected by $Z_{5}$ in step 3 of this round, $i$, while it is detected by $Z_{2}$ in step 2 of round $i+1$, and the corresponding edge is $d_{8}^{i}$ in the $Z$ graph. A $\hat{Z}$ error in an even data qubit is detected in the same way as a $\hat{Z}$ error induced in step 1 .

An $\hat{X}$ error in the measure- $Z$ qubit $Z_{5}$ propagates through $\hat{C}_{Z}(1)$ to $D_{8}$ and $D_{13}$ in steps 3 and 4 as a $\hat{Z}$ error. The $\hat{Z}$ error in $D_{8}$ is detected by $X_{7}$ in step 4 of this round, $i$, while the $\hat{Z}$ error in $D_{13}$ is detected by $X_{8}$ in step 2 of round $i+1$. As a result, the corresponding edge is $c_{5}^{i}$ in the $X$ graph. Similarly to step 1 , a $\hat{Z}$ error corresponds to a detection error, i.e., $v_{5}^{i}$ in the $Z$ graph.

An $\hat{X}$ error in the measure qubit $X_{5}$, similarly to step 1, corresponds to a detection error, i.e., $v_{5}^{i}$ in the $X$ graph. A $\hat{Z}$ error in $X_{5}$ propagates through $\hat{C}_{X}( \pm 1)$ to $D_{12}$ and $D_{13}$ in steps 3 and 4 as an $\hat{X}$ error. The $\hat{X}$ error in $D_{13}$ is detected at $Z_{5}$ in step 4 of this round, $i$, while the $\hat{X}$ error in $D_{12}$ is detected by $Z_{4}$ in step 2 of the following round, $i+1$. As a result, the corresponding edge is $c_{5}^{i}$ in the $Z$ graph.

\section{Step 3}

Below, we consider single-qubit errors induced in the GKP quadrature correction after the third set of two-mode gates in the syndrome measurements (step 3).

An $\hat{X}$ error in an odd data qubit is detected similarly to an $\hat{X}$ error induced in steps 1 and 2. A $\hat{Z}$ error in an odd data qubit, say $D_{13}$, is detected by $X_{8}$ and $X_{5}$ in steps 2 and 3 in the following round, $i+1$, and the corresponding edge is $h_{13}^{i+1}$ in the $X$ graph.

An $\hat{X}$ error in an even data qubit, say $D_{8}$, is detected by $Z_{2}$ and $Z_{5}$ in steps 2 and 3 of the following round, $i+1$, and the corresponding edge is $h_{8}^{i+1}$ in the $Z$ graph. A $\hat{Z}$ error in an even data qubit is detected in the same way as a $\hat{Z}$ error induced in steps 1 and 2 .

An $\hat{X}$ error in the measure- $Z$ qubit $Z_{5}$ propagates through $\hat{C}_{Z}(1)$ to $D_{13}$ in step 4 as a $\hat{Z}$ error and is detected by $X_{8}$ and $X_{5}$ in steps 2 and 3 of the following round, $i+1$. Thus the corresponding edge is $h_{13}^{i+1}$ in the $X$ graph. Similarly to steps 1 and 2 , a $\hat{Z}$ error corresponds to a detection error, i.e., $v_{5}^{i}$ in the $Z$ graph.

An $\hat{X}$ error in the measure qubit $X_{5}$, similarly to steps 1 and 2 , corresponds to a measure, i.e., $v_{5}^{i}$ in the $X$ graph. A $\hat{Z}$ error in $X_{5}$ propagates through $\hat{C}_{X}(1)$ as an $\hat{X}$ error to $D_{12}$ in step 4, where it is detected by $Z_{4}$ and $Z_{8}$ in steps 2 and 3 of the following round, $i+1$. Thus the corresponding edge is $h_{12}^{i+1}$ in the $Z$ graph.

\section{Step 4}

Errors in data qubits $D_{j}$ induced in the GKP quadrature correction after the fourth and final set of two-mode gates in the syndrome measurements (step 4) are detected only in the following round, $i+1$, and the corresponding edges are $h_{j}^{i+1}$ in the $X$ and $Z$ graphs. 
Since measure qubits are now to be measured after this fourth step of the syndrome measurements, they do not go through GKP quadrature correction, and we do not induce any qubit error. Measure- $Z$ qubits are measured in the $\hat{p}$ quadrature, and a wrong measurement outcome (caused by finite squeezing of the GKP spikes in the measured quadrature) corresponds to $v_{j}^{i}$ in the $Z$ graph. Similarly, measure- $X$ qubits are measured in the $\hat{q}$ quadrature, and a wrong measurement outcome corresponds to $v_{j}^{i}$ in the $X$ graph.

\section{Graph boundaries, weighting, and correction}

As described in Secs. III and IV A and in Appendix B, from the residual analog information in the GKP quadrature correction, we can infer the probability of having induced a qubit error during the GKP quadrature correction, which we then use for weighting of the corresponding edges (described above) in the matching graphs. With each edge corresponding to several different errors, the probabilities from multiple GKP quadrature corrections are combined into $p_{\text {tot }}$ by use of Eq. (10) for each edge, and the corresponding edge is finally weighted by $\log _{2}\left(p_{\text {tot }}\right)$.

At the boundary of the code, not all diagonal edges are used. Instead, the corresponding horizontal edge in the following round, $i+1$, is used. As an example, consider an $\hat{X}$ error induced in $D_{3}$ in the code with distance $d=5$ [see Fig. 4(a) for the labeling]. This error is detected by $Z_{2}$ in round $i+1$, but there are no other measure- $Z$ qubits to detect the error in the current round, $i$. Thus, instead of weighting $d_{3}^{i}$, in this case the error probability is included in the weight of $h_{3}^{i+1}$.

In the final round, $i=d+1$, where an ideal syndrome measurement is performed, all data qubits are first corrected with perfect GKP quadrature corrections (using $|\varnothing\rangle_{\mathrm{GKP}}$ states of infinite squeezing) before the stabilizers of the surface code are measured using measure qubits of GKP states with infinite squeezing. As a result, this final round requires only horizontal edges.

After the MWPM decoding, vertices in the matching graphs are matched with error paths following the edges with the most likely errors based on the edge weighting. A vertical edge in an error path, corresponding to a detection error, requires no correction to data qubits. A horizontal or diagonal edge $h_{j}^{i}$ or $d_{j}^{i}$ in an error path in the $Z$ or $X$ graph requires an $\hat{X}$ or $\hat{Z}$ correction, respectively, to data qubit $D_{j}$. Finally, a cross edge $c_{j}^{i}$ in an error path in the $Z$ or $X$ graph requires two $\hat{X}$ or two $\hat{Z}$ corrections, respectively, to neighboring data qubits. As an example, including $c_{5}^{i}$ in Fig. 7(a) in an error path requires $\hat{X}$ corrections to $D_{7}$ and $D_{8}$ or to $D_{12}$ and $D_{13}$. Similarly, including $c_{5}^{i}$ in Fig. 7(b) in an error path requires $\hat{Z}$ corrections to $D_{8}$ and $D_{13}$ or to $D_{9}$ and $D_{14}$.
[1] R. Raussendorf and H. J. Briegel, A One-Way Quantum Computer, Phys. Rev. Lett. 86, 5188 (2001).

[2] N. C. Menicucci, P. van Loock, M. Gu, C. Weedbrook, T. C. Ralph, and M. A. Nielsen, Universal Quantum Computation with Continuous-Variable Cluster States, Phys. Rev. Lett. 97, 110501 (2006).

[3] M. Gu, C. Weedbrook, N. C. Menicucci, T. C. Ralph, and P. van Loock, Quantum computing with continuous-variable clusters, Phys. Rev. A 79, 062318 (2009).

[4] S. Yokoyama, R. Ukai, S. C. Armstrong, C. Sornphiphatphong, T. Kaji, S. Suzuki, J.-i. Yoshikawa, H. Yonezawa, N. C. Menicucci, and A. Furusawa, Ultra-large-scale continuous-variable cluster states multiplexed in the time domain, Nat. Photonics 7, 982 (2013).

[5] M. Chen, N. C. Menicucci, and O. Pfister, Experimental Realization of Multipartite Entanglement of 60 Modes of a Quantum Optical Frequency Comb, Phys. Rev. Lett. 112, 120505 (2014).

[6] J.-i. Yoshikawa, S. Yokoyama, T. Kaji, C. Sornphiphatphong, Y. Shiozawa, K. Makino, and A. Furusawa, Invited article: Generation of one-million-mode continuous-variable cluster state by unlimited time-domain multiplexing, APL Photonics 1, 060801 (2016).

[7] M. V. Larsen, X. Guo, C. R. Breum, J. S. NeergaardNielsen, and U. L. Andersen, Deterministic generation of a two-dimensional cluster state, Science 366, 369 (2019).

[8] W. Asavanant et al., Generation of time-domain-multiplexed two-dimensional cluster state, Science 366, 373 (2019).

[9] W. Asavanant et al., One-hundred step measurement-based quantum computation multiplexed in the time domain with $25 \mathrm{MHz}$ clock frequency, arXiv:2006.11537 [quant-ph] (2020).

[10] M. V. Larsen, X. Guo, C. R. Breum, J. S. NeergaardNielsen, and U. L. Andersen, Deterministic multi-mode gates on a scalable photonic quantum computing platform, Nat. Phys. (2021).

[11] P. T. Cochrane, G. J. Milburn, and W. J. Munro, Macroscopically distinct quantum-superposition states as a bosonic code for amplitude damping, Phys. Rev. A 59, 2631 (1999).

[12] M. H. Michael, M. Silveri, R. T. Brierley, V. V. Albert, J. Salmilehto, L. Jiang, and S. M. Girvin, New Class of Quantum Error-Correcting Codes for a Bosonic Mode, Phys. Rev. X 6, 031006 (2016).

[13] D. Gottesman, A. Kitaev, and J. Preskill, Encoding a qubit in an oscillator, Phys. Rev. A 64, 012310 (2001).

[14] A. Y. Kitaev, Fault-tolerant quantum computation by anyons, Ann. Phys. (Amsterdam) 303, 2 (2003).

[15] R. Raussendorf, J. Harrington, and G. Goyal, A faulttolerant one-way quantum computer, Ann. Phys. (Amsterdam) 321, 2242 (2006).

[16] R. Raussendorf, J. Harrington, and G. Goyal, Topological fault-tolerance in cluster state quantum computation, New J. Phys. 9, 199 (2007).

[17] R. Raussendorf and J. Harrington, Fault-Tolerant Quantum Computation with High Threshold in two Dimensions, Phys. Rev. Lett. 98, 190504 (2007).

[18] K. Fukui, A. Tomita, A. Okamoto, and K. Fujii, HighThreshold Fault-Tolerant Quantum Computation with Ana$\log$ Quantum Error Correction, Phys. Rev. X 8, 021054 (2018). 
[19] B. H. Wu, R. N. Alexander, S. Liu, and Z. Zhang, Quantum computing with multidimensional continuous-variable cluster states in a scalable photonic platform, Phys. Rev. Res. 2, 023138 (2020).

[20] K. Fukui, W. Asavanant, and A. Furusawa, Temporalmode continuous-variable three-dimensional cluster state for topologically protected measurement-based quantum computation, Phys. Rev. A 102, 032614 (2020).

[21] J. E. Bourassa et al., Blueprint for a scalable photonic fault-tolerant quantum computer, Quantum 5, 392 (2021).

[22] X.-C. Yao, T.-X. Wang, H.-Z. Chen, W.-B. Gao, A. G. Fowler, R. Raussendorf, Z.-B. Chen, N.-L. Liu, C.-Y. Lu, Y.-J. Deng, Y.-A. Chen, and J.-W. Pan, Experimental demonstration of topological error correction, Nature 482, 489 (2012).

[23] D. Nigg, M. Müller, E. A. Martinez, P. Schindler, M. Hennrich, T. Monz, M. A. Martin-Delgado, and R. Blatt, Quantum computations on a topologically encoded qubit, Science 345, 302 (2014).

[24] C. K. Andersen, A. Remm, S. Lazar, S. Krinner, N. Lacroix, G. J. Norris, M. Gabureac, C. Eichler, and A. Wallraff, Repeated quantum error detection in a surface code, Nat. Phys. 16, 875 (2020).

[25] A. G. Fowler, M. Mariantoni, J. M. Martinis, and A. N. Cleland, Surface codes: Towards practical large-scale quantum computation, Phys. Rev. A 86, 032324 (2012).

[26] S. B. Bravyi and A. Y. Kitaev, Quantum codes on a lattice with boundary, arXiv:9811052 [quant-ph] (1998).

[27] E. Dennis, A. Kitaev, A. Landahl, and J. Preskill, Topological quantum memory, J. Math. Phys. 43, 4452 (2002).

[28] B. W. Walshe, B. Q. Baragiola, R. N. Alexander, and N. C. Menicucci, Continuous-variable gate teleportation and bosonic-code error correction, Phys. Rev. A 102, 062411 (2020).

[29] K. Noh and C. Chamberland, Fault-tolerant bosonic quantum error correction with the surface-Gottesman-KitaevPreskil code, Phys. Rev. A 101, 012316 (2020).

[30] R. N. Alexander, S. C. Armstrong, R. Ukai, and N. C. Menicucci, Noise analysis of single-mode Gaussian operations using continuous-variable cluster states, Phys. Rev. A 90, 062324 (2014).

[31] M. V. Larsen, J. S. Neergaard-Nielsen, and U. L. Andersen, Architecture and noise analysis of continuous-variable quantum gates using two-dimensional cluster states, Phys. Rev. A 102, 042608 (2020).

[32] P. van Loock, C. Weedbrook, and M. Gu, Building Gaussian cluster states by linear optics, Phys. Rev. A 76, 032321 (2007).

[33] N. C. Menicucci, S. T. Flammia, and P. van Loock, Graphical calculus for Gaussian pure states, Phys. Rev. A 83, 042335 (2011).

[34] We assume the duration and spacing of the temporal modes to be equal. In practice, for a pulsed scheme, the duration of the temporal modes corresponds to the pulse width, while the $\mathrm{nm}$ delay corresponds to a delay of $\mathrm{nm}$ times the temporal pulse spacing.

[35] M. V. Larsen, X. Guo, C. R. Breum, J. S. NeergaardNielsen, and U. L. Andersen, Fiber coupled EPR-state generation using a single temporally multiplexed squeezed light source, npj Quantum Inf. 5, 46 (2019).
[36] S. Takeda, K. Takase, and A. Furusawa, On-demand photonic entanglement synthesizer, Sci. Adv. 5, eaaw4530 (2019).

[37] D. Bonneau, M. Lobino, P. Jiang, C. M. Natarajan, M. G. Tanner, R. H. Hadfield, S. N. Dorenbos, V. Zwiller, M. G. Thompson, and J. L. O'Brien, Fast Path and Polarization Manipulation of Telecom Wavelength Single Photons in Lithium Niobate Waveguide Devices, Phys. Rev. Lett. 108, 053601 (2012).

[38] Y. He et al., Time-Bin-Encoded Boson Sampling with a Single-Photon Device, Phys. Rev. Lett. 118, 190501 (2017).

[39] Compared with Ref. [30], a squeezing operator, dependent on the squeezing of the ancillary $|0\rangle_{\mathrm{sq}}$ states, is missing in Eq. (2). This is because in this work the two-mode entangled states prepared in the resource-preparation gadget are considered as cluster-type states with edge weight 1 [32], similarly to Ref. [10], instead of as approximate cluster states in the language of Ref. [33].

[40] R. Ukai, J.-i. Yoshikawa, N. Iwata, P. van Loock, and A. Furusawa, Universal linear Bogoliubov transformations through one-way quantum computation, Phys. Rev. A 81, 032315 (2010).

[41] V. V. Albert et al., Performance and structure of singlemode bosonic codes, Phys. Rev. A 97, 032346 (2018).

[42] K. Noh, V. V. Albert, and L. Jiang, Quantum capacity bounds of Gaussian thermal loss channels and achievable rates with Gottesman-Kitaev-Preskill codes, IEEE Trans. Inf. Theory 65, 2563 (2019).

[43] J. S. Ivan, K. K. Sabapathy, and R. Simon, Operator-sum representation for bosonic Gaussian channels, Phys. Rev. A 84, 042311 (2011).

[44] R. García-Patrón, C. Navarrete-Benlloch, S. Lloyd, J. H. Shapiro, and N. J. Cerf, Majorization Theory Approach to the Gaussian Channel Minimum Entropy Conjecture, Phys. Rev. Lett. 108, 110505 (2012).

[45] I. Tzitrin, J. E. Bourassa, N. C. Menicucci, and K. K. Sabapathy, Progress towards practical qubit computation using approximate Gottesman-Kitaev-Preskill codes, Phys. Rev. A 101, 032315 (2020).

[46] B. M. Terhal, J. Conrad, and C. Vuillot, Towards scalable bosonic quantum error correction, Quantum Sci. Technol. 5, 043001 (2020).

[47] T. Matsuura, H. Yamasaki, and M. Koashi, Equivalence of approximate Gottesman-Kitaev-Preskill codes, Phys. Rev. A 102, 032408 (2020).

[48] K. Duivenvoorden, B. M. Terhal, and D. Weigand, Singlemode displacement sensor, Phys. Rev. A 95, 012305 (2017).

[49] K. Fukui, A. Tomita, and A. Okamoto, Analog Quantum Error Correction with Encoding a Qubit Into an Oscillator, Phys. Rev. Lett. 119, 180507 (2017).

[50] H. Bombin and M. A. Martin-Delgado, Optimal resources for topological two-dimensional stabilizer codes: Comparative study, Phys. Rev. A 76, 012305 (2007).

[51] Y. Tomita and K. M. Svore, Low-distance surface codes under realistic quantum noise, Phys. Rev. A 90, 062320 (2014).

[52] C. Vuillot, H. Asasi, Y. Wang, L. P. Pryadko, and B. M. Terhal, Quantum error correction with the toric GottesmanKitaev-Preskill code, Phys. Rev. A 99, 032344 (2019). 
[53] J. Edmonds, Paths, trees, and flowers, Can. J. Math. 17, 449 (1965).

[54] J. Edmonds, Maximum matching and a polyhedron with 0, 1 vertices, J. Res. Natl. Bur. Stand., Sect. B 69B, 125 (1965).

[55] E. Knill, Quantum computing with realistically noisy devices, Nature 434, 39 (2005).

[56] D. P. DiVincenzo and P. Aliferis, Effective Fault-Tolerant Quantum Computation with Slow Measurements, Phys. Rev. Lett. 98, 020501 (2007).

[57] B. M. Terhal, Quantum error correction for quantum memories, Rev. Mod. Phys. 87, 307 (2015).

[58] C. Chamberland, P. Iyer, and D. Poulin, Fault-tolerant quantum computing in the Pauli or Clifford frame with slow error diagnostics, Quantum 2, 43 (2018).

[59] C. Flühmann, T. L. Nguyen, M. Marinelli, V. Negnevitsky, K. Mehta, and J. P. Home, Encoding a qubit in a trapped-ion mechanical oscillator, Nature 566, 513 (2019).

[60] P. Campagne-Ibarcq et al., Quantum error correction of a qubit encoded in grid states of an oscillator, Nature 584, $368(2020)$.

[61] B. de Neeve, T. L. Nguyen, T. Behrle, and J. Home, Error correction of a logical grid state qubit by dissipative pumping, arXiv:2010.09681 [quant-ph] (2020).

[62] K. R. Motes, B. Q. Baragiola, A. Gilchrist, and N. C. Menicucci, Encoding qubits into oscillators with atomic ensembles and squeezed light, Phys. Rev. A 95, 053819 (2017).

[63] H. M. Vasconcelos, L. Sanz, and S. Glancy, All-optical generation of states for "Encoding a qubit in an oscillator", Opt. Lett. 34, 3261 (2010).

[64] D. J. Weigand and B. M. Terhal, Generating grid states from Schrödinger-cat states without postselection, Phys. Rev. A 97, 022341 (2018).

[65] M. Eaton, R. Nehra, and O. Pfister, Non-Gaussian and Gottesman-Kitaev-Preskill state preparation by photon catalysis, New J. Phys. 21, 113034 (2019).

[66] Y. Shi, C. Chamberland, and A. Cross, Fault-tolerant preparation of approximate GKP states, New J. Phys. 21, 093007 (2019).

[67] D. Su, C. R. Myers, and K. K. Sabapathy, Conversion of Gaussian states to non-Gaussian states using photonnumber-resolving detectors, Phys. Rev. A 100, 052301 (2019).

[68] A. I. Lvovsky, B. C. Sanders, and W. Tittel, Optical quantum memory, Nat. Photonics 3, 706 (2009).

[69] T. Zhong et al., Nanophotonic rare-earth quantum memory with optically controlled retrieval, Science 357, 1392 (2017).

[70] M. Bouillard, G. Boucher, J. F. Ortas, B. Pointard, and R. Tualle-Brouri, Quantum Storage of Single-Photon and Two-Photon Fock States with an All-Optical Quantum Memory, Phys. Rev. Lett. 122, 210501 (2019).

[71] Y. Hashimoto, T. Toyama, J.-i. Yoshikawa, K. Makino, F. Okamoto, R. Sakakibara, S. Takeda, P. van Loock, and A. Furusawa, All-Optical Storage of Phase-Sensitive
Quantum States of Light, Phys. Rev. Lett. 123, 113603 (2019).

[72] B. W. Walshe, L. J. Mensen, B. Q. Baragiola, and N. C. Menicucci, Robust fault tolerance for continuous-variable cluster states with excess antisqueezing, Phys. Rev. A 100, 010301(R) (2019).

[73] T. Kashiwazaki, N. Takanashi, T. Yamashima, T. Kazama, K. Enbutsu, R. Kasahara, T. Umeki, and A. Furusawa, Continuous-wave 6-dB-squeezed light with $2.5-\mathrm{THz}-$ bandwidth from single-mode PPLN waveguide, APL Photonics 5, 036104 (2020).

[74] N. Takanashi, A. Inoue, T. Kashiwazaki, T. Kazama, K. Enbutsu, R. Kasahara, T. Umeki, and A. Furusawa, Alloptical phase-sensitive detection for ultra-fast quantum computation, Opt. Express 28, 34916 (2020).

[75] J. Wang, F. Sciarrino, A. Laing, and M. G. Thompson, Integrated photonic quantum technologies, Nat. Photonics 14, 273 (2020).

[76] H. J. Kimble, The quantum internet, Nature 453, 1023 (2008).

[77] S. Wehner, D. Elkouss, and R. Hanson, Quantum internet: A vision for the road ahead, Science 362, eaam9288 (2018).

[78] B. Q. Baragiola, G. Pantaleoni, R. N. Alexander, A. Karanjai, and N. C. Menicucci, All-Gaussian Universality and Fault Tolerance with the Gottesman-Kitaev-Preskill Code, Phys. Rev. Lett. 123, 200502 (2019).

[79] H. Yamasaki, T. Matsuura, and M. Koashi, Cost-reduced all-Gaussian universality with the Gottesman-KitaevPreskill code: Resource-theoretic approach to cost analysis, Phys. Rev. Res. 2, 023270 (2020).

[80] J. Hastrup, M. V. Larsen, J. S. Neergaard-Nielsen, N. C. Menicucci, and U. L. Andersen, Cubic phase gates are not suitable for non-Clifford operations on GKP states, Phys. Rev. A 103, 032409 (2021).

[81] C. Horsman, A. G. Fowler, S. Devitt, and R. van Meter, Surface code quantum computing by lattice surgery, New J. Phys. 14, 123011 (2012).

[82] D. Litinski, Magic state distillation: Not as costly as you think, Qauntum 3, 205 (2019).

[83] D. S. Wang, A. G. Fowler, and L. C. L. Hollenberg, Surface code quantum computing with error rates over $1 \%$, Phys. Rev. A 83, 020302(R) (2011).

[84] C. Chamberland, G. Zhu, T. J. Yoder, J. B. Hertzberg, and A. W. Cross, Topological and Subsystem Codes on LowDegree Graphs with Flag Qubits, Phys. Rev. X 10, 011022 (2020).

[85] C. Chamberland, A. Kubica, T. J. Yoder, and G. Zhu, Triangular color codes on trivalent graphs with flag qubits, New J. Phys. 22, 023019 (2020).

[86] C. Chamberland et al., Building a fault-tolerant quantum computer using concatenated cat codes, arXiv:2012.04108 [quant-ph] (2020).

[87] K. Noh, C. Chamberland, and F. G. S. L. Brandão, Low overhead fault-tolerant quantum error correction with the surface-GKP code, arXiv:2103.06994 [quant-ph] (2021). 